\title{
Structure of odd-odd Cs isotopes within the interacting boson-fermion-fermion model based on the Gogny-D1M energy density functional
}

\author{
K. Nomura $\odot,{ }^{1, *}$ R. Rodríguez-Guzmán, ${ }^{2}$ and L. M. Robledo ${ }^{3,4}$ \\ ${ }^{1}$ Department of Physics, Faculty of Science, University of Zagreb, HR-10000 Zagreb, Croatia \\ ${ }^{2}$ Physics Department, Kuwait University, 13060 Kuwait, Kuwait \\ ${ }^{3}$ Departamento de Física Teórica, Universidad Autónoma de Madrid, E-28049 Madrid, Spain \\ ${ }^{4}$ Center for Computational Simulation, Universidad Politécnica de Madrid, Campus de Montegancedo, \\ Boadilla del Monte, E-28660 Madrid, Spain
}

(Received 9 August 2019; revised manuscript received 23 November 2019; published 8 January 2020)

\begin{abstract}
The spectroscopic properties of the odd-odd isotopes ${ }^{124-132} \mathrm{Cs}$ have been studied within the interacting bosonfermion-fermion model based on the Gogny-D1M energy density functional framework. Major ingredients to build the interacting boson-fermion-fermion Hamiltonian, such as the $(\beta, \gamma)$-deformation energy surfaces for the even-even core nuclei ${ }^{124-132} \mathrm{Xe}$ as well as single-particle energies and occupation probabilities of the odd nucleons, have been computed microscopically with the constrained Hartree-Fock-Bogoliubov method. A few coupling constants of the boson-fermion and residual neutron-proton interactions are fitted to reproduce with a reasonable accuracy the experimental excitation energy of the low-lying levels of the odd-mass and odd-odd nuclei. The method is applied to describe the low-energy low-spin spectra of the odd-odd Cs nuclei and the band structures of higher-spin higher-energy states, mainly based on the $\left(v h_{11 / 2}\right)^{-1} \otimes \pi h_{11 / 2}$ configuration. Many of those odd-odd Cs nuclei have been identified as candidates for exhibiting chiral doublet bands.
\end{abstract}

DOI: 10.1103/PhysRevC.101.014306

\section{INTRODUCTION}

A better understanding of the spectroscopic properties of atomic nuclei with an odd number of nucleons still remains a major challenge for both experimental and theoretical lowenergy nuclear physics. The existence of an unpaired nucleon in the nucleus implies the observation of many new effects in nuclear dynamics like the weakening of pairing correlations, the increase of level densities around the Fermi level, polarization of collective degrees of freedom, breaking of time reversal symmetry in the intrinsic wave function, and numerous additional effects. As a consequence, the microscopic description of an odd- $A$ system is far more challenging than in the traditional even-even case [1-3]. This is manifest in the much slower progress in the implementation of symmetry restoration in odd- $A$ nuclei [4,5]. In addition, the quantitative side is strongly affected by tiny details of the nuclear interaction, making this kind of system the perfect test ground to analyze the suitability of new or existing proposals for effective nuclear interactions and functionals; see [6] for a recent analysis focusing on superheavy nuclei. Detailed spectroscopic studies of odd-mass and/or odd-odd nuclei, have already been carried out using microscopic approaches such as the large-scale shell model [7] and the symmetryprojected generator coordinate method (GCM) [4,5]. See [1] for a general introduction to the latter method. From a computational point of view, systematic applications of these

*knomura@phy.hr approaches are very demanding, if not impossible, for heavy nuclei, especially when a large number of valence nucleons are involved and/or multiple shape degrees of freedom have to be taken into account in the generator coordinate method (GCM) ansatz.

To overcome these difficulties we proposed in Ref. [8] (in a study of odd- $A$ Au and $\mathrm{Pt}$ and odd-odd $\mathrm{Au}$ isotopes) to perform constrained Hartree-Fock-Bogoliubov (HFB) calculations based on the Gogny [9] energy density functional (EDF) with the parametrization D1M [10], to obtain energy surfaces as functions of the $(\beta, \gamma)$ quadrupole deformation parameters for the neighboring even-even Pt nuclei. The singleparticle energies and occupation numbers were computed for the odd neutron and odd proton in the odd-mass Au and $\mathrm{Pt}$ as well as odd-odd Au isotopes. Those quantities were then used, as a microscopic input, to completely determine the interacting boson model (IBM) [11] Hamiltonian for the even-even nucleus and most of the parameters of the different boson-fermion coupling terms present in the interacting boson-fermion model (IBFM) [12-14] and the interacting boson-fermion-fermion model (IBFFM) [14,15] Hamiltonians for the odd- $A$ and odd-odd systems, respectively. Only a few coupling constants of the boson-fermion and the residual neutron-proton interaction terms were treated as free parameters. These parameters were determined so as to reproduce reasonably well the experimental low-lying energy levels of the odd-mass and odd-odd nuclei. Though the method involves a few phenomenological parameters, it allows one to study simultaneously the spectroscopy of even-even, oddmass, and odd-odd nuclei within a unified framework. The 
method reduces significantly the computational cost associated with those calculations and provides the possibility of studying heavy odd and odd-odd nuclei irrespective of their location in the chart of nuclides.

In this work, we consider the spectroscopic properties of the odd-odd nuclei ${ }^{124-132} \mathrm{Cs}$, using the theoretical framework developed in Ref. [8]. The reason for the choice of nuclei is that the $A \approx 130$ mass region exhibits a wide variety of structural phenomena. A variety of theoretical models suggested the existence of triaxially deformed and/or $\gamma$-soft shapes for even-even systems in this mass region [16-23]. A gradual transition, from $\gamma$-soft to nearly spherical shapes, has also been identified [24] while several nuclei, such as ${ }^{134} \mathrm{Ba}$ [25] and ${ }^{128} \mathrm{Xe}$ [26], are suggested to display features of the $\mathrm{E}(5)$ critical-point symmetry [27] of the phase transition. In some odd-odd Cs isotopes, most notably in ${ }^{128} \mathrm{Cs}$, chiral doublet bands [28] have been observed [29-32]. Those bands are associated with nearly degenerate energy levels with equal spins and characteristic electromagnetic properties. The highspin level structure in the odd-odd nuclei in the mass $A \approx$ 130 region, in particular the role of the $\left(v h_{11 / 2}\right)^{-1} \otimes \pi h_{11 / 2}$ (neutron hole coupled with proton) or $v h_{11 / 2} \otimes \pi h_{11 / 2}$ configuration in forming the chiral bands, has been studied by various theoretical approaches, and we particularly mention the IBFFM [33] and shell model [34-37] calculations. Furthermore, this mass region represents a challenging testing ground to examine the predictive power of nuclear models for fundamental processes, such as $\beta$ decay and double- $\beta$ decay [38-41]. Previous phenomenological IBFM and IBFFM spectroscopic studies [42] were also carried out for nuclei in the same mass region considered in this work.

The paper is organized as follows. In Sec. II, we outline the theoretical framework used in this study. We begin Sec. III with a brief discussion of the results obtained for the eveneven core nucleus ${ }^{124} \mathrm{Xe}$ as well as the odd- $\mathrm{N}$ and odd- $Z$ nuclei ${ }^{123} \mathrm{Xe}$ and ${ }^{125} \mathrm{Cs}$. In the same section, we discuss the lowenergy spectra obtained for the odd-odd systems ${ }^{124-132}$ Cs. Moreover, we pay attention to the band structures of higherspin states to identify features of chirality in some of the considered odd-odd Cs isotopes. Finally, Sec. IV is devoted to the concluding remarks.

\section{THEORETICAL FRAMEWORK}

\section{A. IBFFM-2 Hamiltonian}

Within the employed theoretical scheme, the low-lying structure of the even-even-core nucleus is described in terms of the IBM [11], where correlated pairs of valence nucleons are represented by bosonic degrees of freedom [43]. In the IBFM, one unpaired nucleon is explicitly included as an additional degree of freedom to the boson space [12-14] to handle odd-mass systems. The IBFFM represents a further extension of the IBFM to odd-odd systems that includes, one unpaired neutron and one unpaired proton $[14,15]$. As in our previous study for odd-odd Au isotopes [8], we have used a version of the IBFFM that distinguishes between neutron and proton degrees of freedom (denoted hereafter as IBFFM-2).
The IBFFM-2 Hamiltonian reads

$$
\hat{H}=\hat{H}_{\mathrm{B}}+\hat{H}_{\mathrm{F}}^{\nu}+\hat{H}_{\mathrm{F}}^{\pi}+\hat{H}_{\mathrm{BF}}^{\nu}+H_{\mathrm{BF}}^{\pi}+\hat{V}_{\mathrm{res}},
$$

where the first term represents the neutron-proton IBM (IBM2) Hamiltonian [43] that describes the even-even core nuclei $\left({ }^{124,126,128,130,132} \mathrm{Xe}\right)$. The second (third) term is the Hamiltonian for an odd neutron (proton). The fourth (fifth) term corresponds to the interaction Hamiltonian describing the coupling of the odd neutron (proton) to the IBM-2 core. The last term in Eq. (1) is the residual interaction between the odd neutron and the odd proton.

For the boson-core Hamiltonian $\hat{H}_{\mathrm{B}}$ in Eq. (1) the standard IBM-2 Hamiltonian has been adopted:

$$
\hat{H}_{\mathrm{B}}=\epsilon\left(\hat{n}_{d_{v}}+\hat{n}_{d_{\pi}}\right)+\kappa \hat{Q}_{v} \cdot \hat{Q}_{\pi},
$$

where $\hat{n}_{d_{\rho}}=d_{\rho}^{\dagger} \cdot \tilde{d}_{\rho} \quad(\rho=v, \pi)$ is the $d$-boson number operator while $\hat{Q}_{\rho}=d_{\rho}^{\dagger} s_{\rho}+s_{\rho}^{\dagger} \tilde{d}_{\rho}^{\dagger}+\chi_{\rho}\left(d_{\rho}^{\dagger} \times \tilde{d}_{\rho}\right)^{(2)}$ is the quadrupole operator. The parameters of the Hamiltonian are $\epsilon, \kappa, \chi_{\nu}$, and $\chi_{\pi}$. The doubly magic nucleus ${ }^{100} \mathrm{Sn}$ is taken as the inert core for the boson space. We have followed the standard way of counting the number of bosons, i.e., the numbers of neutron $N_{v}$ and proton $N_{\pi}$ bosons equal the numbers of neutron-hole and proton-particle pairs, respectively. As a consequence, $N_{\pi}=2$ and $N_{\nu}=6,5,4,3$, and 2 for $124,126,128,130,132 \mathrm{Xe}$, respectively.

In Eq. (1), the Hamiltonian for the odd nucleon, i.e., $\hat{H}_{\mathrm{F}}^{\rho}$ takes the form

$$
\hat{H}_{\mathrm{F}}^{\rho}=-\sum_{j_{\rho}} \epsilon_{j_{\rho}} \sqrt{2 j_{\rho}+1}\left(a_{j_{\rho}}^{\dagger} \times \tilde{a}_{j_{\rho}}\right)^{(0)},
$$

where $\epsilon_{j_{v}}\left(\epsilon_{j_{\pi}}\right)$ and $j_{v}\left(j_{\pi}\right)$ stand for the single-particle energy and the angular momentum of the unpaired neutron (proton). On the other hand, $a_{j_{\rho}}^{(\dagger)}\left(a_{j_{\rho}}\right)$ represents the fermion creation (annihilation) operator while $\tilde{a}_{j_{\rho}}$ is defined as $\tilde{a}_{j m}=$ $(-1)^{j-m} a_{j-m}$. For the fermion valence space, we have taken into account the full neutron and proton major shells $N, Z=$ $50-82$, that include the $3 s_{1 / 2}, 2 d_{3 / 2}, 2 d_{5 / 2}, 1 g_{7 / 2}$, and $1 h_{11 / 2}$ orbitals.

For the boson-fermion interaction term, $\hat{H}_{\mathrm{BF}}^{\rho}$ in Eq. (1), we employ the form that has been formulated within a simple generalized seniority scheme $[13,14]$ :

$$
\hat{H}_{\mathrm{BF}}^{\rho}=\Gamma_{\rho} \hat{Q}_{\rho^{\prime}} \cdot \hat{q}_{\rho}+\Lambda_{\rho} \hat{V}_{\rho^{\prime} \rho}+A_{\rho} \hat{n}_{d_{\rho}} \hat{n}_{\rho},
$$

where $\rho^{\prime} \neq \rho$, and the first, second, and third terms are the quadrupole dynamical, exchange, and monopole terms, respectively. The strength parameters of the interaction Hamiltonian are denoted by $\Gamma_{\rho}, \Lambda_{\rho}$, and $A_{\rho}$. As in previous studies $[13,44]$, we have assumed that both the dynamical and exchange terms are dominated by the interaction between unlike particles, i.e., between the odd neutron and proton bosons and between the odd proton and neutron bosons. We also assume that for the monopole term the interaction between like particles, i.e., between the odd neutron and neutron bosons and between the odd proton and proton bosons, plays a dominant role. In Eq. (4), $\hat{Q}_{\rho}$ is the bosonic quadrupole operator identical to the one in the IBM-2 Hamiltonian in Eq. (2) with the same value of the parameter $\chi_{\rho}$. The fermionic quadrupole 
operator $\hat{q}_{\rho}$ reads

$$
\hat{q}_{\rho}=\sum_{j_{\rho} j_{\rho}^{\prime}} \gamma_{j_{\rho} j_{\rho}^{\prime}}\left(a_{j_{\rho}}^{+} \times \tilde{a}_{j_{\rho}^{\prime}}\right)^{(2)}
$$

where $\quad \gamma_{j_{\rho} j_{\rho}^{\prime}}=\left(u_{j_{\rho}} u_{j_{\rho}^{\prime}}-v_{j_{\rho}} v_{j_{\rho}^{\prime}}\right) Q_{j_{\rho} j_{\rho}^{\prime}} \quad$ and $\quad Q_{j_{\rho} j_{\rho}^{\prime}}=$ $\left\langle l \frac{1}{2} j_{\rho} \| Y^{(2)}|| l^{\prime} \frac{1}{2} j_{\rho}^{\prime}\right\rangle \quad$ represents the matrix element of the fermionic quadrupole operator in the considered single-particle basis. The exchange term $\hat{V}_{\rho^{\prime} \rho}$ in Eq. (4) reads

$$
\begin{aligned}
\hat{V}_{\rho^{\prime} \rho}= & -\left(s_{\rho^{\prime}}^{+} \tilde{d}_{\rho^{\prime}}\right)^{(2)} \cdot\left\{\sum_{j_{\rho} j_{\rho}^{\prime} j_{\rho}^{\prime \prime}} \sqrt{\frac{10}{N_{\rho}\left(2 j_{\rho}+1\right)}} \beta_{j_{\rho} j_{\rho}^{\prime}} \beta_{j_{\rho}^{\prime \prime} j_{\rho}}\right. \\
& \left.\times:\left(\left(d_{\rho}^{+} \times \tilde{a}_{j_{\rho}^{\prime \prime}}\right)^{\left(j_{\rho}\right)} \times\left(a_{j_{\rho}^{\prime}}^{+} \times \tilde{s}_{\rho}\right)^{\left(j_{\rho}^{\prime}\right)}\right)^{(2)}:\right\}+(\text { H.c. }),
\end{aligned}
$$

with $\beta_{j_{\rho} j_{\rho}^{\prime}}=\left(u_{j_{\rho}} v_{j_{\rho}^{\prime}}+v_{j_{\rho}} u_{j_{\rho}^{\prime}}\right) Q_{j_{\rho} j_{\rho}^{\prime}}$. In the second line of the above equation the standard notation: ( $\cdots)$ : indicates normal ordering. For the monopole term, the number operator for the odd fermion is expressed as $\hat{n}_{\rho}=\sum_{j_{\rho}}\left(-\sqrt{2 j_{\rho}+1}\right)\left(a_{j_{\rho}}^{+} \times\right.$ $\left.\tilde{a}_{j_{\rho}}\right)^{(0)}$.

Finally, we adopted the following form of the residual neutron-proton interaction $\hat{V}_{\text {res }}$ :

$$
\begin{aligned}
\hat{V}_{\text {res }}= & 4 \pi u_{\mathrm{D}} \delta\left(\mathbf{r}_{v}-\mathbf{r}_{\pi}\right) \\
& +u_{\mathrm{T}}\left\{\frac{3\left(\sigma_{\nu} \cdot \mathbf{r}_{v \pi}\right)\left(\sigma_{\pi} \cdot \mathbf{r}_{v \pi}\right)}{r_{v \pi}^{2}}-\sigma_{v} \cdot \sigma_{\pi}\right\},
\end{aligned}
$$

where the first and second terms denote the delta and tensor interactions, respectively. We have found that these two terms are enough to provide a reasonable description of the lowlying states in the considered odd-odd nuclei. Note that by definition $\mathbf{r}_{v \pi}=\mathbf{r}_{v}-\mathbf{r}_{\pi}$ and that $u_{\mathrm{D}}$ and $u_{\mathrm{T}}$ are the parameters of this term. Furthermore, the matrix element $V_{\text {res }}^{\prime}$ of the residual interaction $\hat{V}_{\text {res }}$ can be expressed as [45]

$$
\begin{aligned}
V_{\text {res }}^{\prime}= & \left(u_{j_{v}^{\prime}} u_{j_{\pi}^{\prime}} u_{j_{v}} u_{j_{v}}+v_{j_{v}^{\prime}} v_{j_{\pi}^{\prime}} v_{j_{v}} v_{j_{v}}\right) V_{j_{j_{j}^{\prime}}^{\prime} j_{\pi}^{\prime} j_{v} j_{\pi}}^{J} \\
& -\left(u_{j_{v}^{\prime}} v_{j_{\pi}^{\prime}} u_{j_{v}} v_{j_{\pi}}+v_{j_{v}^{\prime}} u_{j_{\pi}^{\prime}} v_{j_{v}} u_{j_{\pi}}\right) \\
& \times \sum_{J^{\prime}}\left(2 J^{\prime}+1\right)\left\{\begin{array}{lll}
j_{v}^{\prime} & j_{\pi} & J^{\prime} \\
j_{v} & j_{\pi}^{\prime} & J
\end{array}\right\} V_{j_{v}^{\prime} j_{\pi} j_{v} j_{\pi}^{\prime}}^{J^{\prime}},
\end{aligned}
$$

where

$$
V_{j_{v}^{\prime} j_{\pi}^{\prime} j_{v} j_{\pi}}^{J}=\left\langle j_{v}^{\prime} j_{\pi}^{\prime} ; J\left|\hat{V}_{\text {res }}\right| j_{v} j_{\pi} ; J\right\rangle
$$

represents the matrix element between the neutron-proton pairs and $J$ stands for the total angular momentum of the neutron-proton pair. The bracket in Eq. (8) represents the corresponding Racah coefficient. The terms resulting from contractions are neglected in Eq. (8), as in Ref. [46].

\section{B. Procedure to build the IBFFM-2 Hamiltonian}

The basic ingredients of the IBFFM-2 Hamiltonian $\hat{H}$ in Eq. (1) are determined as follows [8]:

(1) Once the form of the IBM-2 Hamiltonian is fixed, the parameters $\epsilon, \kappa, \chi_{\nu}$, and $\chi_{\pi}$ are uniquely determined
TABLE I. Parameters of the IBM-2 Hamiltonian $\hat{H}_{\mathrm{B}}$ for the eveneven isotopes ${ }^{124-132} \mathrm{Xe}$.

\begin{tabular}{lcccc}
\hline \hline & $\epsilon(\mathrm{MeV})$ & $\kappa(\mathrm{MeV})$ & $\chi_{\nu}$ & $\chi_{\pi}$ \\
\hline${ }^{124} \mathrm{Xe}$ & 0.45 & -0.336 & 0.40 & -0.50 \\
${ }^{126} \mathrm{Xe}$ & 0.52 & -0.323 & 0.25 & -0.50 \\
${ }^{128} \mathrm{Xe}$ & 0.62 & -0.315 & 0.25 & -0.55 \\
${ }^{130} \mathrm{Xe}$ & 0.82 & -0.308 & 0.38 & -0.50 \\
${ }^{132} \mathrm{Xe}$ & 0.90 & -0.250 & 0.20 & -0.55 \\
\hline \hline
\end{tabular}

[47,48] by mapping the $(\beta, \gamma)$-deformation energy surface obtained from the constrained Gogny-D1M [10] HFB calculation onto the expectation value of the IBM-2 Hamiltonian in the boson coherent state [49].

(2) The single-neutron Hamiltonian $\hat{H}_{\mathrm{F}}^{v}$ and the bosonfermion Hamiltonian $\hat{H}_{\mathrm{BF}}^{v}$ for odd- $N$ Xe isotopes are built by using the procedure of [50] (see also [51] for further details). In those references, the single-particle energies and occupation probabilities of the odd nucleon, entering both $\hat{H}_{\mathrm{F}}^{v}$ and $\hat{H}_{\mathrm{BF}}^{v}$, are obtained from Gogny-D1M HFB calculations at zero deformation. The optimal values of the boson-fermion interaction strengths $\Gamma_{\nu}, \Lambda_{\nu}$, and $A_{v}$ in Eq. (4), are chosen, separately for positive and negative parity, so as to reproduce with a reasonable accuracy the experimental low-energy levels of each odd- $N$ Xe nucleus. A similar procedure has been employed to determine the parameters $\Gamma_{\pi}, \Lambda_{\pi}$, and $A_{\pi}$ for the odd- $Z$ Cs isotopes.

(3) We use for the IBFFM-2 Hamiltonian in the odd-odd Cs the same strength parameters $\Gamma_{v}, \Lambda_{v}$, and $A_{v}\left(\Gamma_{\pi}\right.$, $\Lambda_{\pi}$, and $A_{\pi}$ ) obtained for the odd- $N$ Xe (odd- $Z$ Cs) nuclei in the previous step. The single-particle energies and occupation probabilities are, however, computed independently for each of the studied odd-odd systems.

(4) Finally, the parameters $u_{\mathrm{D}}$ and $u_{\mathrm{T}}$, in the residual interaction $\hat{V}_{\text {res }}$, are determined so as to reproduce with reasonable accuracy the low-lying spectra in the odd-odd nuclei under consideration. For simplicity, we have taken the fixed values $u_{\mathrm{D}}=0.7 \mathrm{MeV}$ and $u_{\mathrm{T}}=0.02 \mathrm{MeV}$ for all the considered nuclei and for both parities.

The values of the IBM-2 parameters adopted for the eveneven Xe isotopes are shown in Table I. In particular, the sum $\chi_{v}+\chi_{\pi}$ is somewhat close to zero in many of the considered $\mathrm{Xe}$ isotopes. This indicates that these nuclei are close to the $\mathrm{O}(6)$ limit of the IBM, which is associated with $\gamma$-soft deformation.

The fitted strength parameters of the boson-fermion interactions, $\hat{H}_{\mathrm{BF}}^{\rho}$, are shown in Table II. The values of some of these strength parameters, i.e., $\Gamma_{\rho}$ and $\Lambda_{\rho}$, for a given configuration ( $s d g$ or $h_{11 / 2}$ ) gradually change with neutron number. For the positive-parity states in ${ }^{128,130,132} \mathrm{Cs}$, the values of $\Gamma_{\pi}$ for the proton $h_{11 / 2}$ configuration (which are fitted to the oddmass nuclei ${ }^{129,131,133} \mathrm{Cs}$, respectively) have been modified so that the higher-spin positive-parity states, which are mainly 
TABLE II. Parameters for the boson-fermion coupling Hamiltonians $\hat{H}_{\mathrm{B} F}^{v}$ and $\hat{H}_{\mathrm{B} F}^{\pi}$ (in $\mathrm{MeV}$ ). These values have been adopted for describing the odd-odd nuclei ${ }^{124-132} \mathrm{Cs}$. For the positive-parity states in ${ }^{128,130,132} \mathrm{Cs}$, the values of the parameter $\Gamma_{\pi}$ for the $h_{11 / 2}$ orbital are different compared to those employed for the negative-parity states and are shown in parentheses.

\begin{tabular}{lccccccl}
\hline \hline & & $\Gamma_{\nu}$ & $\Lambda_{v}$ & \multicolumn{1}{c}{$A_{v}$} & \multicolumn{1}{c}{$\Gamma_{\pi}$} & $\Lambda_{\pi}$ & \multicolumn{1}{c}{$A_{\pi}$} \\
\hline${ }^{124} \mathrm{Cs}$ & $s d g$ & 3.20 & 0.20 & -0.14 & 0.80 & 0.51 & -0.80 \\
& $h_{11 / 2}$ & 3.20 & 4.80 & -0.20 & 0.60 & 0.51 & -2.2 \\
${ }^{126} \mathrm{Cs}$ & $s d g$ & 3.00 & 0.40 & -0.12 & 0.80 & 0.40 & -0.70 \\
& $h_{11 / 2}$ & 3.00 & 1.85 & 0.00 & 1.00 & 0.50 & -1.0 \\
${ }^{128} \mathrm{Cs}$ & $s d g$ & 3.00 & 0.60 & -0.28 & 1.00 & 0.40 & -0.70 \\
& $h_{11 / 2}$ & 3.00 & 1.33 & 0.00 & $1.00(2.60)$ & 0.50 & -1.3 \\
${ }^{130} \mathrm{Cs}$ & $s d g$ & 1.60 & 2.20 & -0.30 & 1.20 & 0.55 & -0.80 \\
& $h_{11 / 2}$ & 1.60 & 0.92 & -0.48 & $1.20(2.40)$ & 0.55 & -1.3 \\
${ }^{132} \mathrm{Cs}$ & $s d g$ & 1.00 & 2.00 & -0.30 & 1.20 & 0.58 & -0.50 \\
& $h_{11 / 2}$ & 1.00 & 0.95 & -0.34 & $1.20(3.00)$ & 0.58 & -0.55 \\
\hline \hline
\end{tabular}

composed of the $\left(v h_{11 / 2}\right)^{-1} \otimes \pi h_{11 / 2}$ configuration, become lower in energy. We consider a value of $\approx 0.5 \mathrm{MeV}$ for the excitation energy $E_{\mathrm{x}}$. The modified $\Gamma_{\pi}$ values, given in parentheses in Table II, are also different from those employed for the negative-parity states.

Finally, the single-particle energies and occupation probabilities for the odd-odd $\mathrm{Cs}$ isotopes, obtained using the Gogny-D1M HFB approach, are given in Table III. They are quite similar to the ones obtained in the case of the odd- $N$ Xe and odd- $Z$ Cs nuclei.

Once the value of all the parameters has been obtained, the IBFFM-2 Hamiltonian is diagonalized in the $\left|L_{v} L_{\pi}(L) ; j_{v} j_{\pi}(J): I\right\rangle$ basis characterized by the angular momentum $L_{v}\left(L_{\pi}\right)$ of the neutron (proton) bosons, the total angular momentum $L$ for the even-even boson core, and the total angular momentum $I$ of the coupled system.

\section{Transition operators}

Using the wave functions obtained after the diagonalization of the IBFFM-2 Hamiltonian, the electric quadrupole $(E 2)$ and magnetic dipole $(M 1)$ properties can be computed. The corresponding $\hat{T}^{(E 2)}$ and $\hat{T}^{(M 1)}$ operators are given by [8]

$$
\begin{aligned}
\hat{T}^{(E 2)}= & e_{\nu}^{B} \hat{Q}_{\nu}+e_{\pi}^{B} \hat{Q}_{\pi}-\frac{1}{\sqrt{5}} \sum_{\rho=\nu, \pi} \sum_{j_{\rho} j_{\rho}^{\prime}} \\
& \times\left(u_{j_{\rho}} u_{j_{\rho}^{\prime}}-v_{j_{\rho}} v_{j_{\rho}^{\prime}}\right)\left\langle j_{\rho}^{\prime}|| e_{\rho}^{F} r^{2} Y^{(2)}|| j_{\rho}\right\rangle\left(a_{j_{\rho}}^{\dagger} \times \tilde{a}_{j_{\rho}^{\prime}}\right)^{(2)},
\end{aligned}
$$

and

$$
\begin{aligned}
\hat{T}^{(M 1)}= & \sqrt{\frac{3}{4 \pi}}\left\{g_{v}^{B} \hat{L}_{v}^{B}+g_{\pi}^{B} \hat{L}_{\pi}^{B}-\frac{1}{\sqrt{3}} \sum_{\rho=v, \pi} \sum_{j j^{\prime}}\right. \\
& \left.\times\left(u_{j_{\rho}} u_{j_{\rho}^{\prime}}+v_{j_{\rho}} v_{j_{\rho}^{\prime}}\right)\left\langle j_{\rho}^{\prime}|| g_{l}^{\rho} \mathbf{I}+g_{s}^{\rho} \mathbf{s}|| j_{\rho}\right\rangle\left(a_{j_{\rho}}^{\dagger} \times \tilde{a}_{j_{\rho}^{\prime}}\right)^{(1)}\right\} .
\end{aligned}
$$

In Eq. (10), $e_{\rho}^{B}$ and $e_{\rho}^{F}$ are the effective charges for the boson and fermion systems. We have employed the fixed values $e_{v}^{B}=$ $e_{\pi}^{B}=0.15 \mathrm{eb}$, and $e_{v}^{F}=0.5 \mathrm{eb}$ and $e_{\pi}^{F}=1.5 \mathrm{eb}$. In the case of the $M 1$ operator in Eq. (11), $g_{v}^{B}$ and $g_{\pi}^{B}$ are $g$ factors for the neutron and proton bosons. We have also used the fixed values $g_{v}^{B}=0 \mu_{N}$ and $g_{\pi}^{B}=1.0 \mu_{N}$ [11,52]. For the neutron (proton) $g$ factors, the usual Schmidt values $g_{l}^{v}=0 \mu_{N}$ and $g_{s}^{v}=-3.82 \mu_{N}\left(g_{l}^{\pi}=1.0 \mu_{N}\right.$ and $\left.g_{s}^{\pi}=5.58 \mu_{N}\right)$ have been considered. Both the proton and neutron $g_{s}$ values have been quenched $30 \%$.

\section{RESULTS AND DISCUSSION}

In this section, we will briefly discuss some selected results obtained for even-even Xe and odd-mass Cs nuclei. The nuclei ${ }^{124} \mathrm{Xe}$ (Sec. III A), ${ }^{123} \mathrm{Xe}$ and ${ }^{125} \mathrm{Cs}$ (Sec. III B) will be taken as representative examples. As we are mainly interested in the structure of odd-odd nuclei, most of our discussions will be devoted to the spectroscopic results obtained for such odd-odd systems (Sec. III C).

\section{A. Even-even nuclei}

In Ref. [54], we have considered transitions from $\gamma$-soft to nearly spherical shapes in the even-even isotopes ${ }^{126-136} \mathrm{Xe}$ as well as in the case of odd-mass Xe and Cs nuclei. The same

TABLE III. Neutron and proton single-particle energies (in MeV) and occupation probabilities for the odd-odd Cs isotopes.

\begin{tabular}{ccccccccccccc}
\hline \hline \multirow{2}{*}{${ }^{124} \mathrm{Cs}$} & $3 s_{1 / 2}$ & $2 d_{3 / 2}$ & $2 d_{5 / 2}$ & $1 g_{7 / 2}$ & $1 h_{11 / 2}$ & & $3 s_{1 / 2}$ & $2 d_{3 / 2}$ & $2 d_{5 / 2}$ & $1 g_{7 / 2}$ & $1 h_{11 / 2}$ \\
& $\epsilon_{j_{v}}$ & 1.339 & 1.003 & 3.719 & 3.439 & 0.000 & $\epsilon_{j_{\pi}}$ & 2.555 & 2.476 & 0.122 & 0.000 & 3.674 \\
${ }^{126} \mathrm{Cs}$ & $v_{j_{v}}^{2}$ & 0.602 & 0.506 & 0.929 & 0.902 & 0.243 & $v_{j_{\pi}}^{2}$ & 0.034 & 0.047 & 0.303 & 0.352 & 0.023 \\
& $\epsilon_{j_{v}}$ & 1.271 & 0.983 & 3.684 & 3.516 & 0.000 & $\epsilon_{j_{\pi}}$ & 2.680 & 2.525 & 0.207 & 0.000 & 3.674 \\
& $v_{j_{v}}^{2}$ & 0.692 & 0.618 & 0.944 & 0.925 & 0.332 & $v_{j_{\pi}}^{2}$ & 0.032 & 0.047 & 0.290 & 0.362 & 0.024 \\
${ }^{128} \mathrm{Cs}$ & $\epsilon_{j_{v}}$ & 1.217 & 0.978 & 3.656 & 3.607 & 0.000 & $\epsilon_{j_{\pi}}$ & 2.809 & 2.580 & 0.298 & 0.000 & 3.668 \\
& $v_{j_{v}}^{2}$ & 0.770 & 0.718 & 0.956 & 0.943 & 0.431 & $v_{j_{\pi}}^{2}$ & 0.030 & 0.046 & 0.276 & 0.373 & 0.024 \\
${ }^{130} \mathrm{Cs}$ & $\epsilon_{j_{v}}$ & 1.174 & 0.984 & 3.635 & 3.710 & 0.000 & $\epsilon_{j_{\pi}}$ & 2.942 & 2.642 & 0.392 & 0.000 & 3.655 \\
& $v_{j_{v}}^{2}$ & 0.838 & 0.805 & 0.968 & 0.958 & 0.541 & $v_{j_{\pi}}^{2}$ & 0.028 & 0.045 & 0.261 & 0.384 & 0.025 \\
${ }^{132} \mathrm{Cs}$ & $\epsilon_{j_{v}}$ & 1.141 & 1.001 & 3.620 & 3.823 & 0.000 & $\epsilon_{j_{\pi}}$ & 3.081 & 2.712 & 0.5 & 0.000 & 3.637 \\
& $v_{j_{v}}^{2}$ & 0.896 & 0.878 & 0.977 & 0.972 & 0.660 & $v_{j_{\pi}}^{2}$ & 0.026 & 0.044 & 0.246 & 0.395 & 0.025 \\
\hline \hline
\end{tabular}



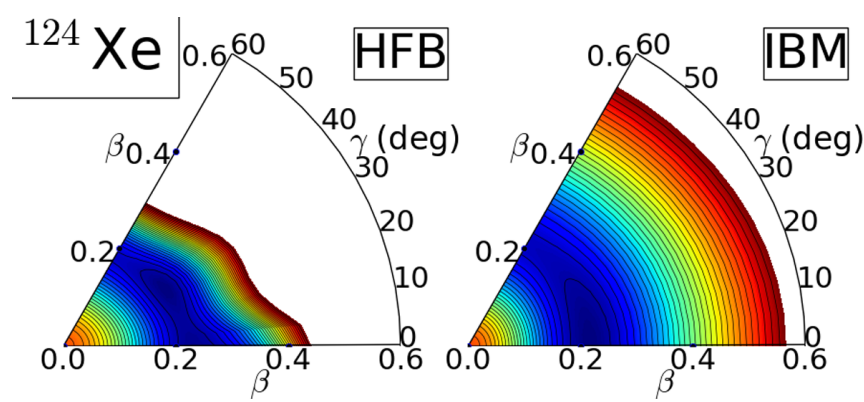

FIG. 1. The Gogny-D1M and IBM-2 $(\beta, \gamma)$-deformation energy surfaces obtained for ${ }^{124} \mathrm{Xe}$ are plotted up to $3 \mathrm{MeV}$ from the global minimum. The energy difference between neighboring contours is $100 \mathrm{keV}$.

Gogny-D1M energy surfaces for ${ }^{126-132}$ Xe used in that work have been used to fix, this time, the parameters of the IBM-2 Hamiltonian for these nuclei. Only the energy surface of ${ }^{124} \mathrm{Xe}$ has been added to the results obtained in previous calculations. A major difference with respect to Ref. [54] is that now we use the IBFM-2 instead of the IBFM-1 model, which does not distinguish between neutron and proton bosons. Another minor difference with respect to Ref. [54] is that now the eveneven ${ }^{A+1} \mathrm{Xe}_{N+1}$ nucleus is taken as a reference to obtain the results for the odd- $N$ isotope ${ }^{A} \mathrm{Xe}_{N}$.

The Gogny-D1M and the (mapped) IBM-2 energy surfaces obtained for ${ }^{124} \mathrm{Xe}$ are depicted in Fig. 1. The HFB energy surface exhibits a shallow triaxial minimum with $\gamma \approx 30^{\circ}$. Such a triaxial minimum can only be obtained in the IBM-2 after including higher-order (e.g., three-body) terms. We are, however, neglecting such higher-order terms in this study because of the lack of IBFFM and IBFM computer codes

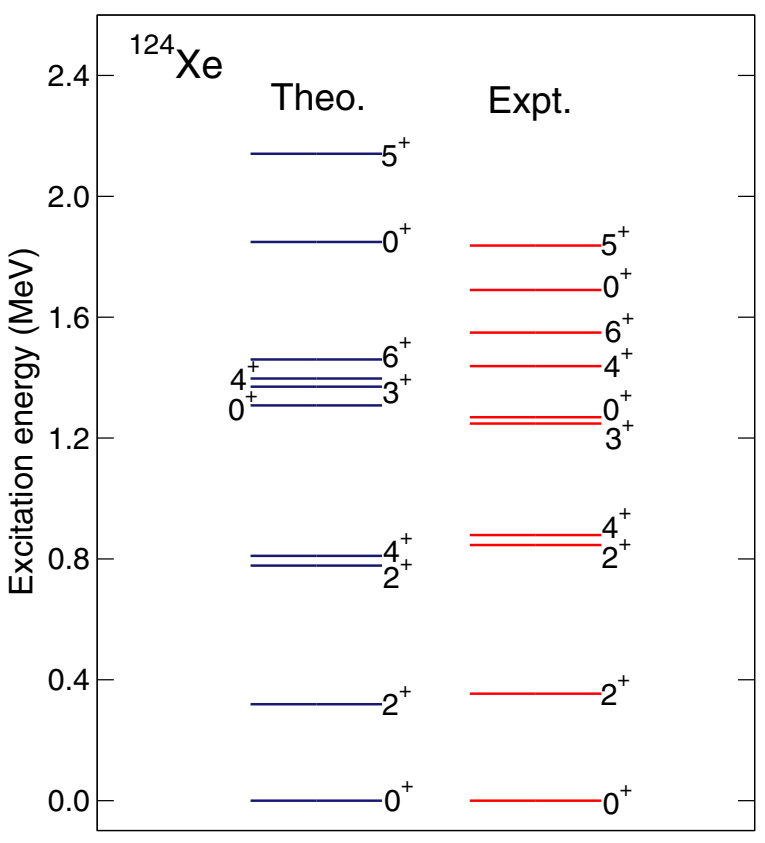

FIG. 2. Theoretical and experimental [53] low-energy excitation spectra for ${ }^{124} \mathrm{Xe}$.

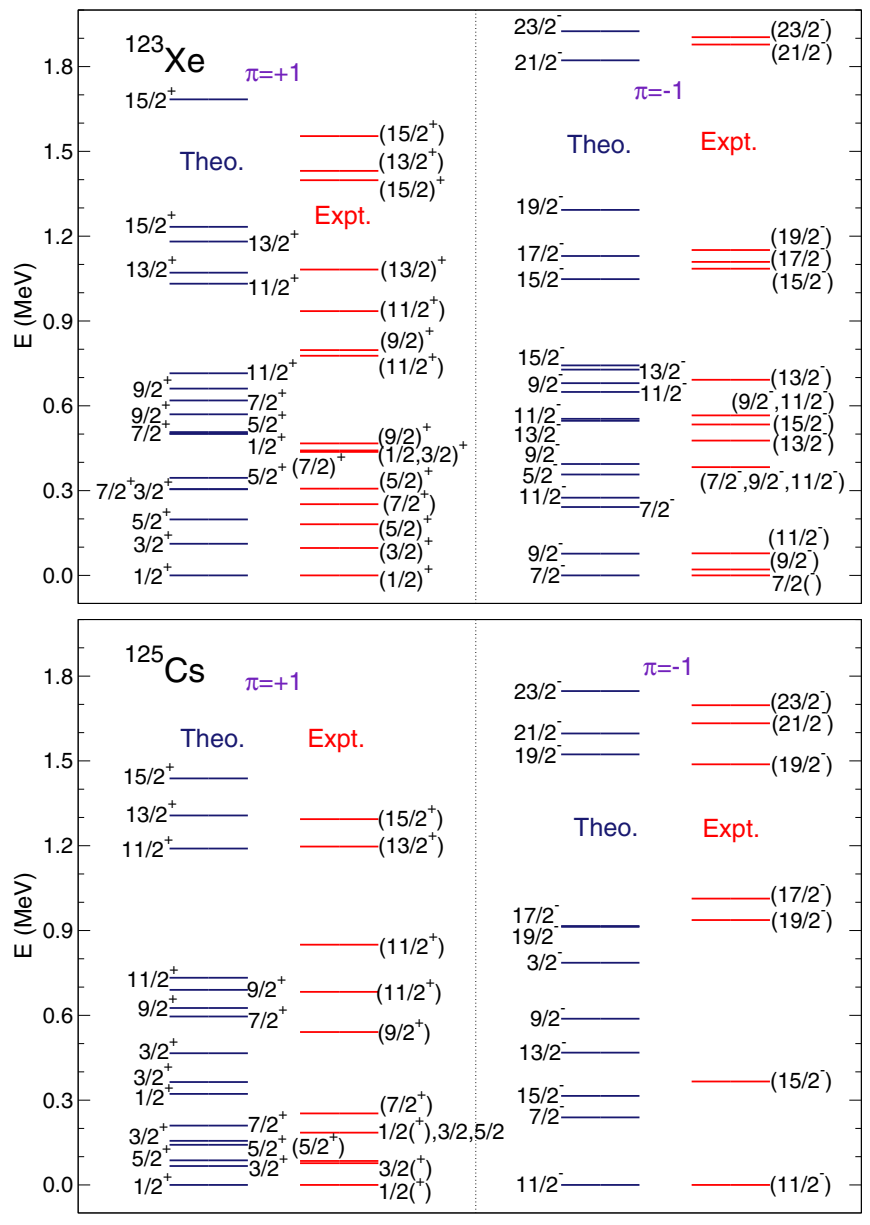

FIG. 3. Same as Fig. 2, but for the odd-N ${ }^{123} \mathrm{Xe}$ and odd-Z ${ }^{125} \mathrm{Cs}$ nuclei. The spin and/or parity in parentheses have not been established experimentally.

able to handle them. As seen in Fig. 1, the IBM-2 surface is much flatter than the HFB far away from the global mean-field minimum. This is a consequence of the reduced IBM model space and it has already been found and discussed in great detail in our previous studies $[47,48]$. These are not serious limitations as the most relevant configurations for the study of low-lying collective states are those around the global minimum and we have paid special attention to reproduce them.

The energy spectrum provided by the IBM-2 Hamiltonian for ${ }^{124} \mathrm{Xe}$ is compared in Fig. 2 with the experimental data [53]. As can be seen, our calculations reproduce well the experimental spectrum without any phenomenological adjustment. Both the theoretical and experimental spectra exhibit features resembling those of the $\mathrm{O}(6)$ dynamical symmetry, i.e., $R_{4 / 2}=E\left(4_{1}^{+}\right) / E\left(2_{1}^{+}\right) \approx 2.5$, a low-lying $2_{2}^{+}$level close to the $4_{1}^{+}$one, and the nearly staggered energy systematic of the $\gamma$ band [i.e., $2_{2}^{+},\left(3_{1}^{+}, 4_{2}^{+}\right),\left(5_{1}^{+}, 6_{2}^{+}\right)$, etc $)$.

\section{B. Odd-mass nuclei}

Let us turn our attention to the nuclei ${ }^{123} \mathrm{Xe}$ and ${ }^{125} \mathrm{Cs}$. The low-lying positive- and negative-parity states obtained for 


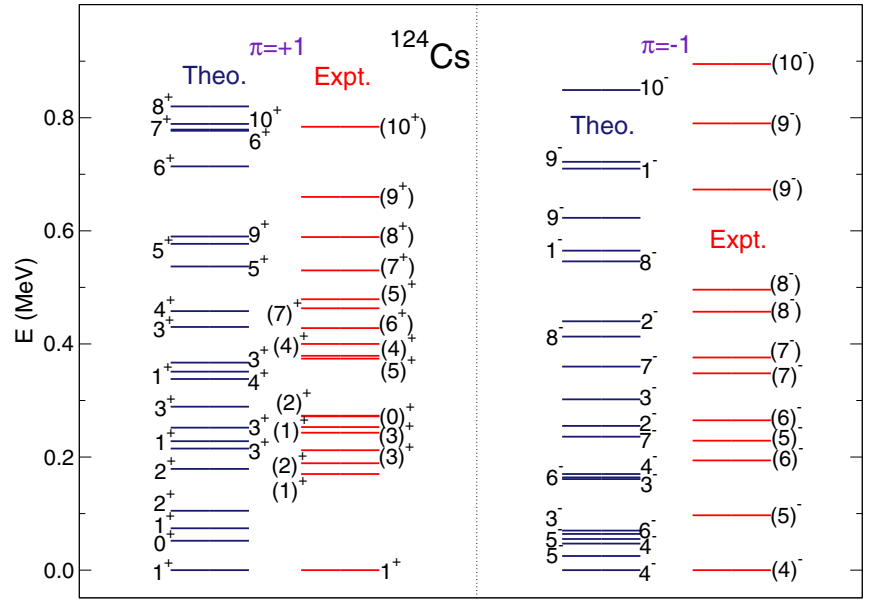

FIG. 4. Low-lying positive- and negative-states of the odd-odd nucleus ${ }^{124}$ Cs. Experimental energy levels are taken from Ref. [53].

those nuclei are shown in Fig. 3. They are compared with the available experimental data [53]. Our results suggest that the low-lying positive-parity states in ${ }^{123} \mathrm{Xe}$ are mainly built via the coupling of the odd neutron hole in the $3 s_{1 / 2}$ and $2 d_{3 / 2}$ single-particle orbitals to the even-even boson core $\left({ }^{124} \mathrm{Xe}\right)$. On the other hand, the negative-parity states are accounted for by the unique-parity $1 h_{11 / 2}$ single-particle configuration. As seen in Fig. 3, our results agree well with the experiment for both parities. In the case of ${ }^{125} \mathrm{Cs}$, the low-lying positive-parity states are mainly based on the $1 g_{7 / 2}$ and $2 d_{5 / 2}$ single-particle configurations. In the lower panels of Fig. 3 a reasonable agreement between the predicted IBFM-2 and the experimental spectra is observed.

\section{Odd-odd Cs isotopes}

\section{Energy spectra for the low-spin low-energy states}

Let us now discuss the results obtained for odd-odd Cs nuclei. We will consider low-spin low-energy states up to an excitation energy $E_{\mathrm{x}} \approx 1 \mathrm{MeV}$. Our calculation indicates that those states are mainly based on normal-parity (i.e., $s d g$ ) orbitals.

The spectra obtained for ${ }^{124,126,128,130,132} \mathrm{Cs}$ are depicted in Figs. 4-8, respectively. In the case of ${ }^{124} \mathrm{Cs}$ (see Fig. 4), the predicted positive- and negative-parity states agree well with the experimental ones. The IBFFM-2 wave function of the $1_{1}^{+}$ground state is composed of the mixture of several single-particle configurations, among which the largest (about $50 \%$ ) contribution comes from the odd neutron hole in the $3 s_{1 / 2}$ orbital. As for the negative-parity states, the predicted IBFFM-2 wave functions for the lowest $4_{1}^{-}, 5_{1}^{-}$, and $6_{1}^{-}$states are complex mixtures of different single-particle configurations. In those states, the neutron $v h_{11 / 2}$ coupled to the proton in either the $3 s_{1 / 2}, 2 d_{3 / 2}, 2 d_{5 / 2}$, or $1 g_{7 / 2}$ positive-parity orbital plays a dominant role.

In the case of ${ }^{126} \mathrm{Cs}$ (see Fig. 5), the agreement with the experiment is as good as for ${ }^{124} \mathrm{Cs}$. The structure of the wave functions corresponding to the lowest positive-parity states is similar to the one obtained for ${ }^{124} \mathrm{Cs}$ [i.e., they are

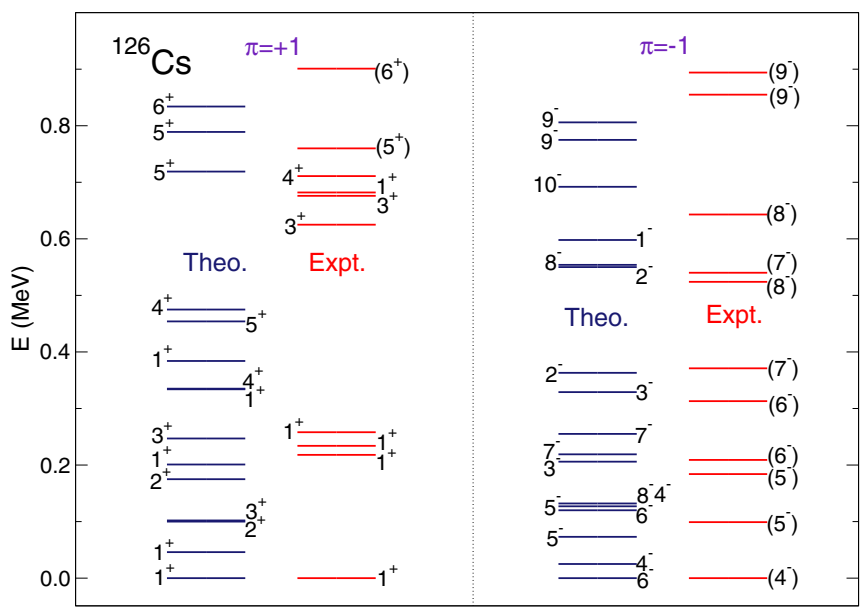

FIG. 5. Same as in Fig. 4 but for ${ }^{126}$ Cs. Experimental data for positive- and negative-parity states are taken from Refs. [53,55], respectively.

mainly accounted for by the $\left(v s_{1 / 2}\right)^{-1} \otimes \pi s d g$ configuration]. In our calculation the lowest-energy negative-parity state is predicted to be the $6_{1}^{-}$one. The main component $(47 \%)$ of the IBFFM-2 wave function of this $6_{1}^{-}$state is the configuration $\left[\left(v h_{11 / 2}\right)^{-1} \otimes \pi g_{7 / 2}\right]^{\left(J=8^{-}\right)}$. Experimentally, the $4^{-}$state is suggested to be the lowest negative-parity state, and the tentative $6_{1}^{-}$level is found at a much higher excitation energy than in our calculation. However, for most of the low-lying negative-parity states both spin and parity have not been firmly established.

The experimental data are more scarce for the ${ }^{128} \mathrm{Cs}$ nucleus, as well as for the heavier ones ${ }^{130,132} \mathrm{Cs}$. For the nucleus ${ }^{128} \mathrm{Cs}$, experimental information is only available for a couple of $1^{+}$states. Here, we stress that our calculations reproduce the correct ground-state spin $I=1_{1}^{+}$. Note, that the predicted $1_{2}^{+}$and $1_{3}^{+}$non-yrast states are found below $200 \mathrm{keV}$ excitation energy, somewhat similarly to the experimental situation. Furthermore, we also obtain $2^{+}$and $3^{+}$states below $200 \mathrm{keV}$. The structure of the $1_{1}^{+}, 2_{1}^{+}$, and $3_{1}^{+}$wave functions is similar

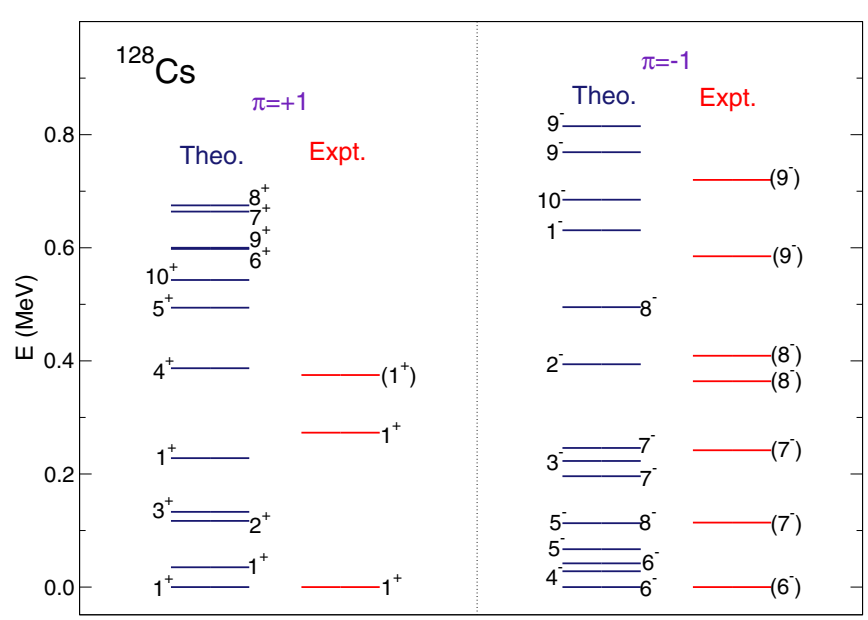

FIG. 6. Same as in Fig. 4 but for ${ }^{128}$ Cs. 


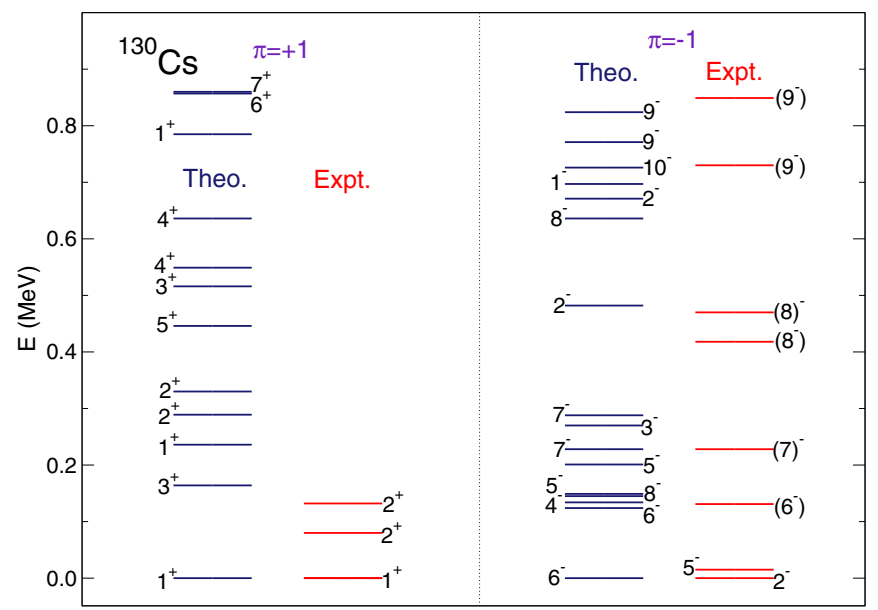

FIG. 7. Same as in Fig. 4 but for ${ }^{130}$ Cs.

to the one in ${ }^{124} \mathrm{Cs}$ and ${ }^{126} \mathrm{Cs}$. Concerning the negative-parity states of ${ }^{128} \mathrm{Cs}$, the predicted low-spin levels are in reasonable agreement with the experimental ones. However, our calculations suggest several states near the ground state, that have not been observed experimentally (i.e., a $4^{-}$and two $5^{-}$states, and the second $6^{-}$state).

The positive-parity low-spin spectrum obtained for ${ }^{130} \mathrm{Cs}$ is shown in Fig. 7. Once more, our calculations predict the correct ground-state spin $I=1^{+}$. However, the two experimental $2^{+}$states around $100 \mathrm{keV}$ excitation energy are overestimated by a factor of 3 . This is not surprising as the excitation energy of levels is often overestimated within the IBM framework, for those nuclei near a shell closure, and the reason is the decreasing number of active bosons. This also seems to be the case for both ${ }^{130} \mathrm{Cs}$ and ${ }^{132} \mathrm{Cs}$. In addition, the structure of the IBFFM-2 wave function corresponding to the $1_{1}^{+}$state turns out to be slightly different than the ground states of the lighter odd-odd systems ${ }^{124-128}$ Cs. The contribution of the $v d_{3 / 2}$ single-particle configuration becomes larger in ${ }^{130,132} \mathrm{Cs}$ than in ${ }^{124-128} \mathrm{Cs}$. The HFB deformation energy surfaces obtained for even-even Xe isotopes [54] exhibit a structural
TABLE IV. Theoretical and experimental quadrupole $Q(I)$ (in $e \mathrm{~b}$ units) and magnetic $\mu(I)$ (in $\mu_{N}$ units) moments for ${ }^{124-132}$ Cs. The experimental values are taken from Ref. [56].

\begin{tabular}{llll}
\hline \hline & & Theory & Experiment \\
\hline${ }^{124} \mathrm{Cs}$ & $Q\left(1_{1}^{+}\right)$ & -0.475 & $-0.74(3)$ \\
& $\mu\left(1_{1}^{+}\right)$ & +0.377 & $+0.673(3)$ \\
${ }^{126} \mathrm{Cs}$ & $Q\left(1_{1}^{+}\right)$ & -0.585 & $-0.68(2)$ \\
& $\mu\left(1_{1}^{+}\right)$ & +0.869 & $+0.777(4)$ \\
${ }^{128} \mathrm{Cs}$ & $Q\left(1_{1}^{+}\right)$ & -0.471 & $-0.570(8)$ \\
& $\mu\left(1_{1}^{+}\right)$ & +0.794 & $+0.974(5)$ \\
${ }^{130} \mathrm{Cs}$ & $Q\left(1_{1}^{+}\right)$ & -0.125 & $-0.059(6)$ \\
& $\mu\left(1_{1}^{+}\right)$ & +0.573 & $+1.460(7)$ \\
& $Q\left(5_{1}^{-}\right)$ & -0.314 & $+1.45(5)$ \\
& $\mu\left(5_{1}^{-}\right)$ & -1.062 & $+0.629(4)$ \\
${ }^{132} \mathrm{Cs}$ & $Q\left(2_{1}^{+}\right)$ & -0.062 & $+0.508(7)$ \\
& $\mu\left(2_{1}^{+}\right)$ & +0.940 & $+2.222(7)$ \\
\hline \hline
\end{tabular}

change from ${ }^{128} \mathrm{Xe}$ ( $\gamma$-soft shape with a shallow triaxial minimum) to ${ }^{130} \mathrm{Xe}$ (nearly spherical shape with a shallow prolate minimum). Such a structural change in the even-even systems seems to be more or less translated into the structure of the IBFFM-2 wave functions of the odd-odd systems. As can be seen from Fig. 7, the disagreement with the experimental data is more pronounced for negative-parity states. The energies of the $2_{1}^{-}$and $5_{1}^{-}$levels, which are suggested to be the lowest negative-parity states experimentally, are, however, too high in our calculations.

Finally, the positive- and negative-parity low-spin lowenergy spectra obtained for ${ }^{132} \mathrm{Cs}$ are depicted in Fig. 8. Here, the comparison with the experiment is worse, but one should keep in mind that this nucleus is the closest to the $N=82$ shell closure. As a result, the number of neutron $N_{v}=2$ and proton $N_{\pi}=2$ bosons is probably not enough for a detailed description of the level structure in the framework of the IBM. Other possible reasons are first that the single-particle energies and occupation probabilities for odd nucleons, obtained from the Gogny-D1M calculation, may not be realistic enough in this

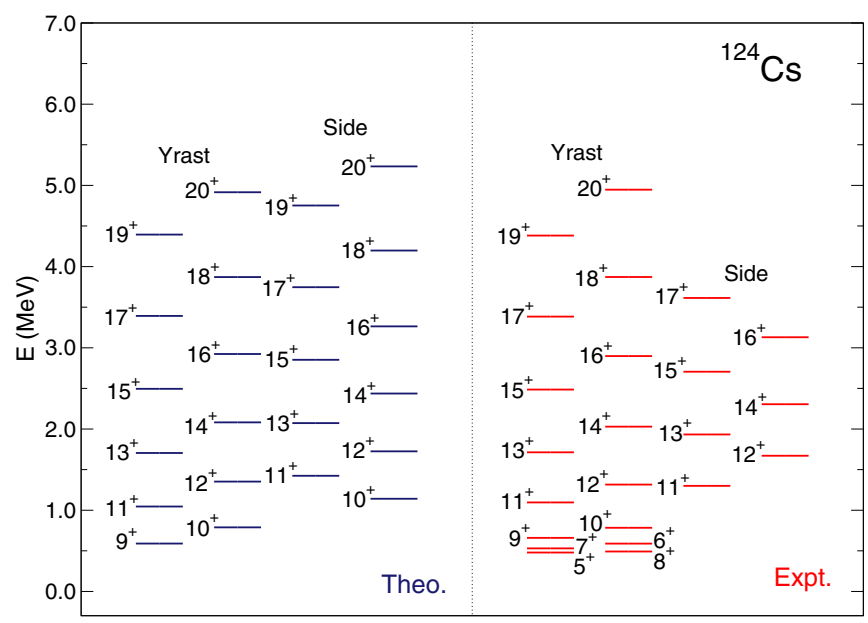

FIG. 9. Band structure of the higher-spin higher-energy positiveparity states in ${ }^{124} \mathrm{Cs}$.

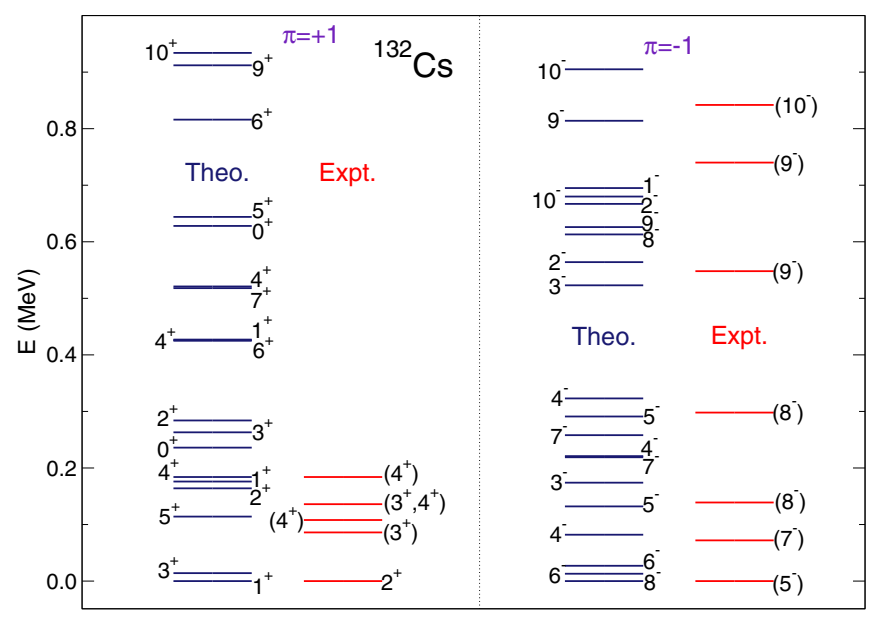

FIG. 8. Same as in Fig. 4 but for ${ }^{132}$ Cs. 


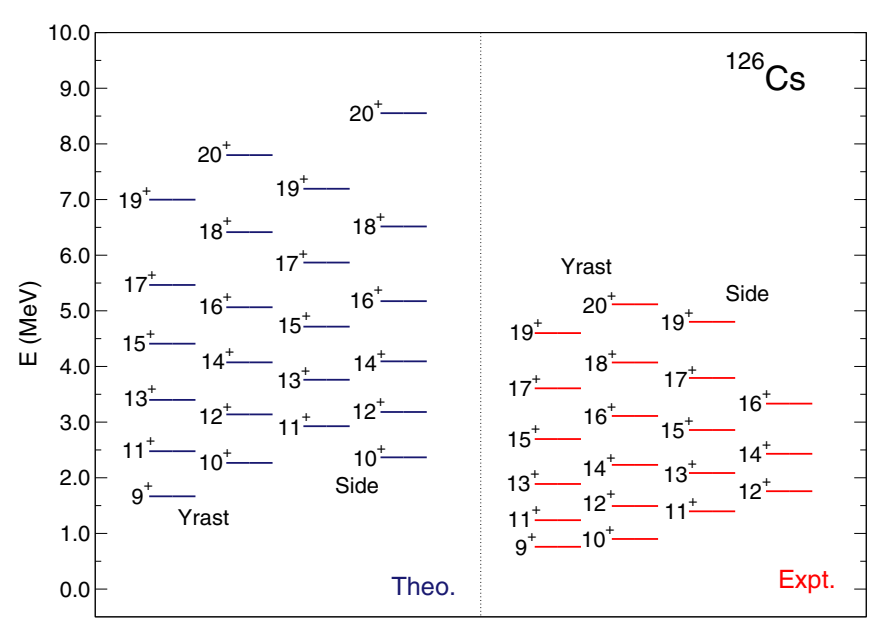

FIG. 10. Same as in Fig. 9 but for ${ }^{126}$ Cs.

case. Finally, the fixed values of the strengths and/or the forms of the residual neutron-proton interactions employed in the IBFFM-2 Hamiltonian are too restrictive.

\section{E2 and M1 moments of lowest-lying states}

As for the electromagnetic properties of the lowest-lying states in odd-odd Cs isotopes, experimental data are only available for the quadrupole $Q(I)$ and magnetic dipole $\mu(I)$ moments. The theoretical and the available experimental $Q(I)$ and $\mu(I)$ values are compared in Table IV. For the ${ }^{124,126,128} \mathrm{Cs}$ nuclei, the predicted $Q(I)$ and $\mu(I)$ moments agree well with the experimental ones, in both magnitude and sign. However, some of the moments obtained for some states in ${ }^{130,132} \mathrm{Cs}$ are opposite in sign to their experimental counterparts. This corroborates that the energy levels of the corresponding states in these nuclei have not been described well with respect to the experimental data (see Figs. 7 and 8), and could have occurred because of the assumption of using the fixed strength

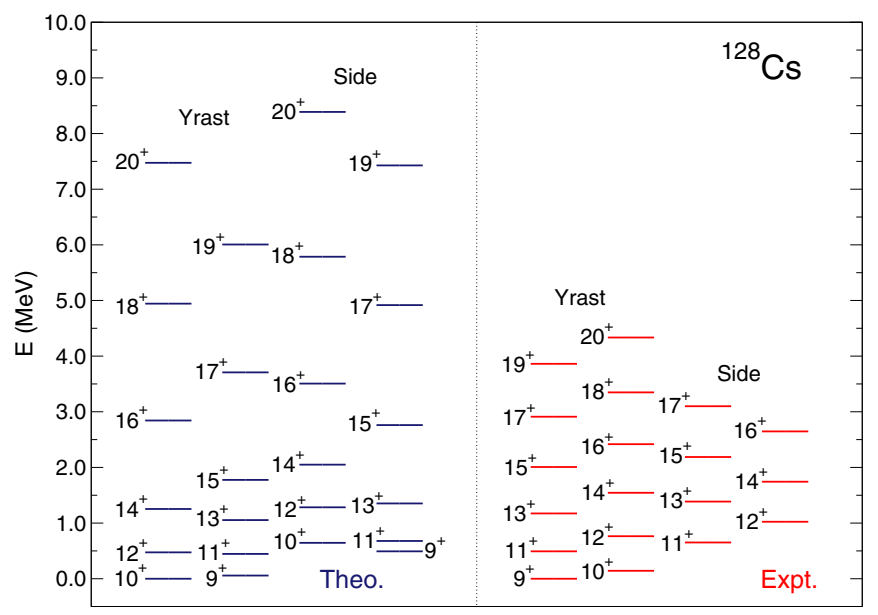

FIG. 11. Same as in Fig. 9 but for ${ }^{128}$ Cs. The theoretical and experimental spectra are normalized with respect to the $10_{1}^{+}$and $9_{1}^{+}$states, respectively, which are the lowest states based on the $\left(v h_{11 / 2}\right)^{-1} \otimes \pi h_{11 / 2}$ configuration.

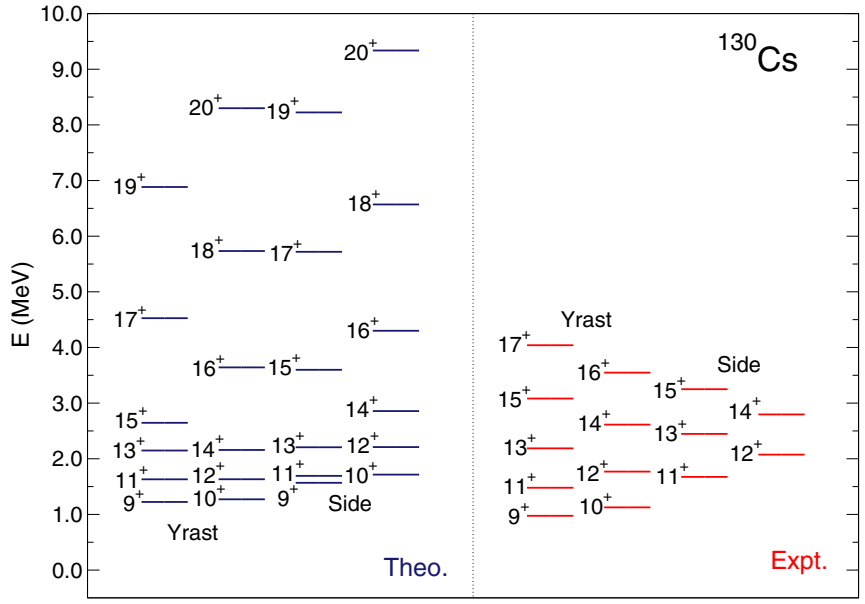

FIG. 12. Same as in Fig. 9 but for ${ }^{130}$ Cs.

parameters for the residual neutron-proton interaction $\hat{V}_{\text {res }}$ and/or, again, because of the more restricted configuration space for the boson system.

\section{Band structure of higher-spin states}

We have further studied the detailed band structure of the higher-lying higher-spin states in the considered odd-odd Cs isotopes. We have paid special attention to the possible doublet structure expected as a result of the coupling between a neutron hole and a proton in the unique-parity $1 h_{11 / 2}$ orbital. Our calculations suggest that the higher-spin states in most of the considered odd-odd Cs nuclei are almost entirely composed of $\left[\left(\nu h_{11 / 2}\right)^{-1} \otimes \pi h_{11 / 2}\right]^{(J)}$ neutron-proton pairs coupled to the even-even boson core, as expected empirically.

The high-spin bands predicted for the nuclei $124,126,128,130,132 \mathrm{Cs}$ are depicted from Figs. 9 to 13 , respectively. In each of these figures, for both theoretical and experimental states, the two bands on the left-hand side with the $\Delta I=1$ level sequence, and the other two bands

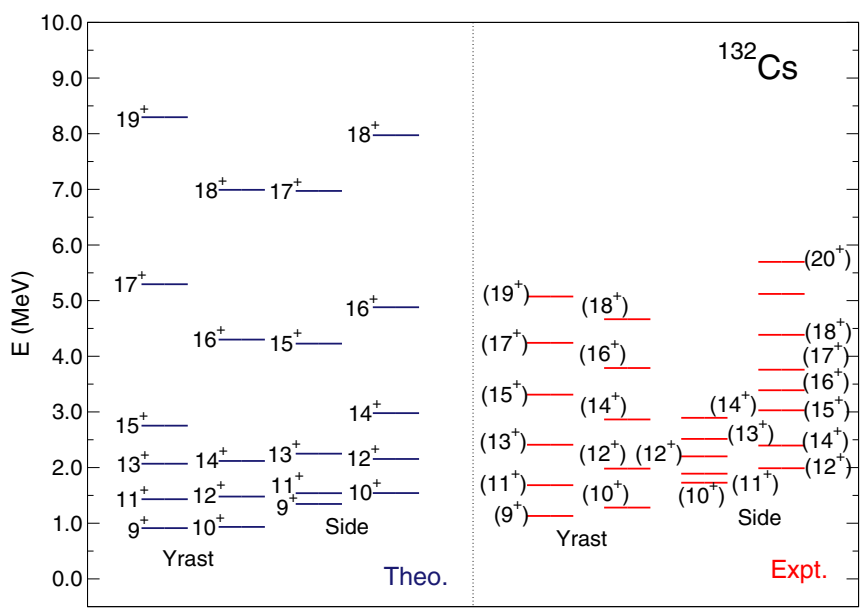

FIG. 13. Same as in Fig. 9 but for ${ }^{132}$ Cs. For the experimental level between the $18^{+}$and $20^{+}$states in the side band, even the tentative spin and parity are not known [53]. 

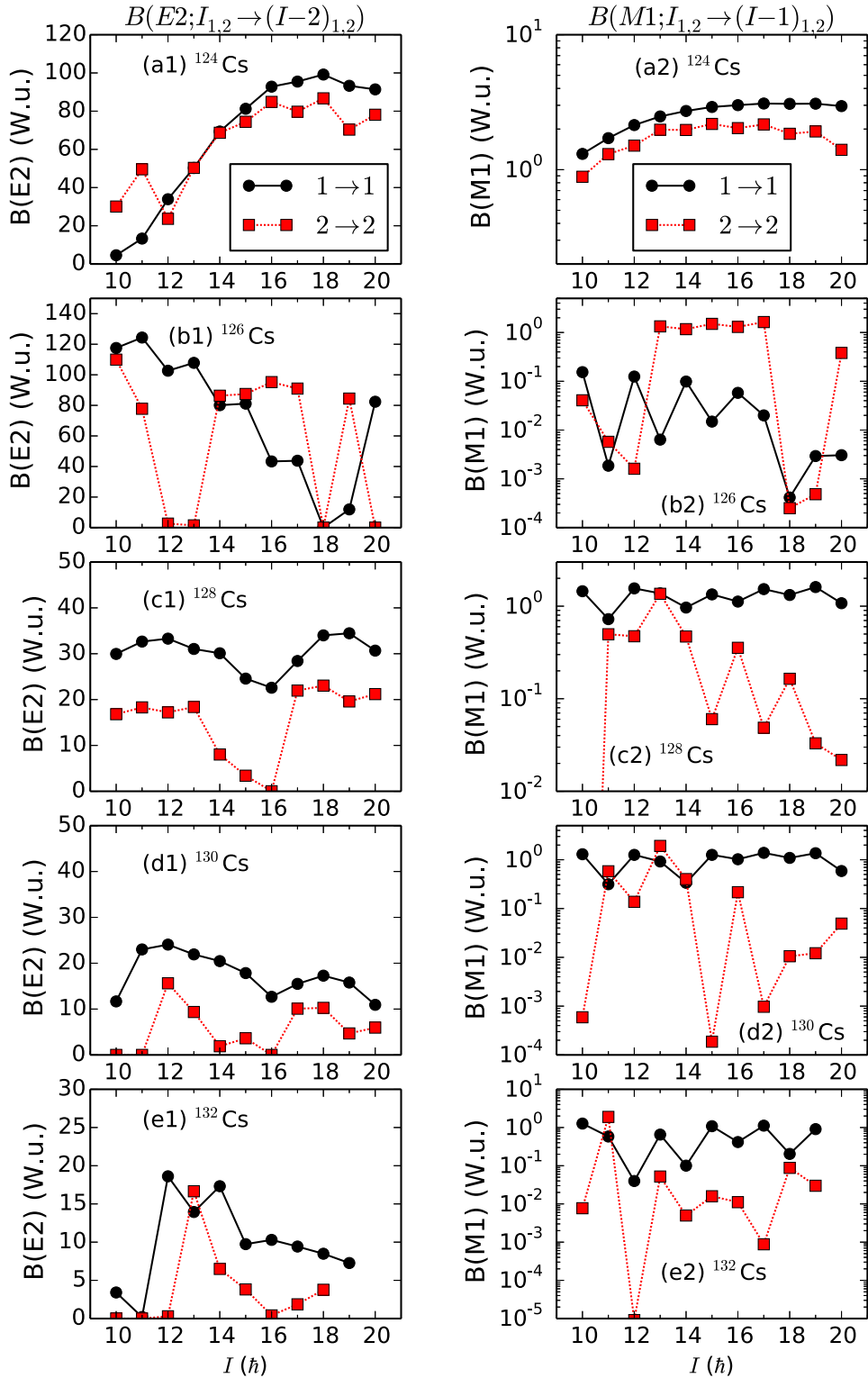
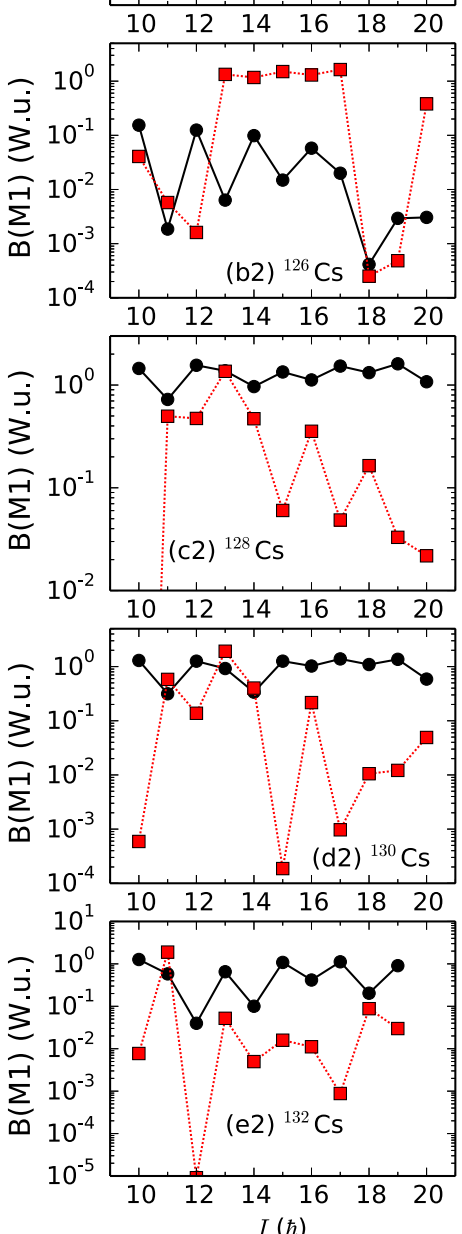
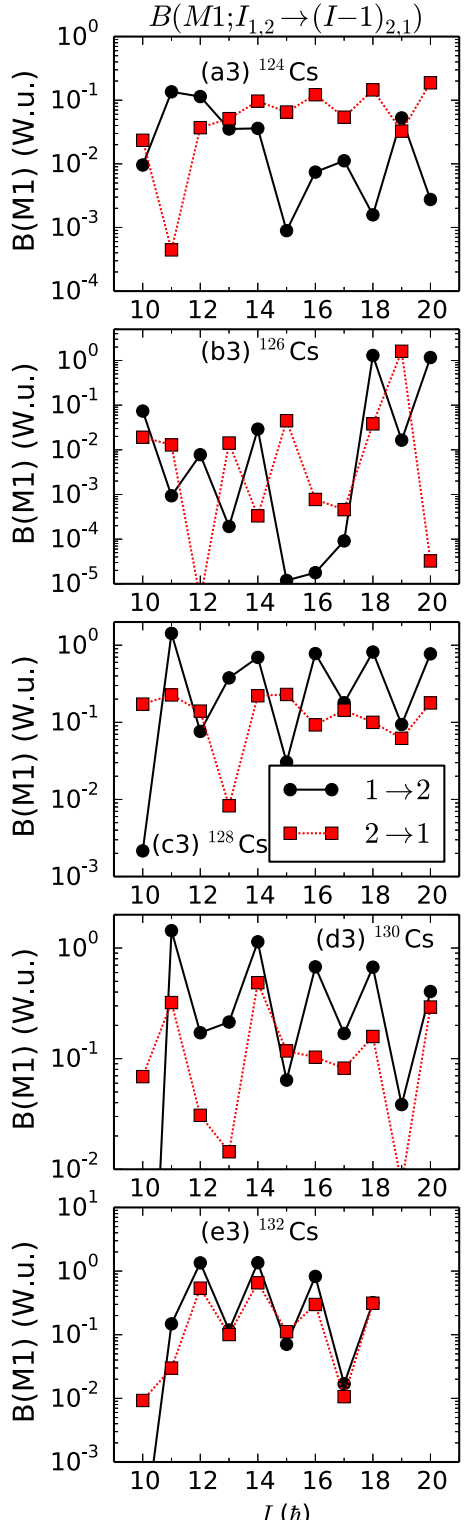

FIG. 14. The calculated $B(E 2 ; I \rightarrow I-2)$ and $B(M 1 ; I \rightarrow I-1)$ transition strengths (in Weisskopf units) for the positive-parity bands of the ${ }^{124-132} \mathrm{Cs}$ nuclei. Left column [panels (a1) to (e1)]: the intraband $B\left(E 2 ; I_{1,2} \rightarrow(I-2)_{1,2}\right)$ transition rates between the yrast states $\left[I_{1}\right.$ and $\left.(I-2)_{1}\right]$ and between the second lowest states $\left[I_{2}\right.$ and $\left.(I-2)_{2}\right]$ with a given spin $I$. Middle column [panels (a2) to (e2)]: the intraband $B\left(M 1 ; I_{1,2} \rightarrow(I-1)_{1,2}\right)$ transition strengths. Right column [panels (a3) to (e3)]: the interband $B\left(M 1 ; I_{1,2} \rightarrow(I-1)_{2,1}\right)$ transition strengths.

on the right-hand side with the $\Delta I=1$ level sequence are identified as the yrast and side bands, respectively. As for the theoretical bands for each nucleus, we have simply grouped the calculated states $I_{1}$ (the lowest states with spin $I$ ) and $I_{2}$ (the second lowest states with spin $I$ ) into the yrast and side bands, respectively.

In the case of ${ }^{124} \mathrm{Cs}$ (see Fig. 9) the experimental band structure is well reproduced, including the energies of the bandhead states. However, for ${ }^{126} \mathrm{Cs}$ (see Fig. 10) the bandhead energies of the experimental bands are overestimated by a factor of around 2. Also, because of the limited size of the boson space as the $N=82$ shell closure is approached the theoretical bands look more stretched than the experimentally identified ones as the spin increases. Nevertheless, for
${ }^{126} \mathrm{Cs}$ the overall structure of the theoretical spectrum agrees reasonably well with the experimental one. The calculated higher-spin bands for ${ }^{126} \mathrm{Cs}$ shown in Fig. 10 resemble well the doublet-like bands, i.e., close-lying states with the same spin $I$. However, the main components of the wave functions of the yrast states up to $I \leqslant 17^{+}$are coming from the coupling between the odd neutron and odd proton in the normal-parity $s d g$ orbitals, not the $\left[\left(\nu h_{11 / 2}\right)^{-1} \otimes \pi h_{11 / 2}\right]^{(J)}$ neutron-proton pair configurations as in all the other odd-odd Cs nuclei considered.

As for ${ }^{128} \mathrm{Cs}$, the absolute energies of the observed bands have not been established experimentally. Therefore, in Fig. 11 both the experimental and calculated energy levels for ${ }^{128} \mathrm{Cs}$ are plotted with respect to the experimental $10_{1}^{+}$state, 
which is suggested to be the bandhead of the lowest-energy band based on the $\left[\left(v h_{11 / 2}\right)^{-1} \otimes \pi h_{11 / 2}\right]^{(J)}$ configuration. In general, the structures of the bands identified experimentally are well reproduced up to $I \approx 16^{+}$. However, the energy of higher-spin states is overestimated. With increasing spin the stretching of the predicted bands, as compared with the experiment, becomes larger than in ${ }^{126} \mathrm{Cs}$.

In Figs. 12 and 13, similar doublet-like band structures are obtained also in ${ }^{130,132} \mathrm{Cs}$ and are in a good agreement with the experimental spectra up to relatively low spin, e.g., $I \leqslant 15^{+}$. The moments of inertia for higher-spin states are considerably underestimated due to the fact that the configuration space of the even-even boson core becomes much smaller for those nuclei close to the neutron shell closure $N=82$. For instance, in ${ }^{132} \mathrm{Cs}$ there is only one $19^{+}$state, which is formed by the configuration where four $d$ bosons and a neutron and a proton in the $h_{11 / 2}$ orbital are all aligned, i.e., $L=8$ and $\left[\left(v h_{11 / 2}\right)^{-1} \otimes \pi h_{11 / 2}\right]^{(11)}$. Also there is no state with spin higher than $I=19^{+}$. Possible solutions to improve the description of the higher-spin states in the IBFFM-2 can be, for instance, the inclusions of an additional boson degree of freedom, e.g., $L=4^{+}(\mathrm{g})$ boson, and of higher quasiparticle excitations or broken pairs. These extensions are, however, out of the scope of the present study.

\section{4. $B(E 2)$ and $B(M 1)$ systematic in the high-spin states}

To identify possible signatures of chirality we have considered, in addition to energy levels, the systematic of the $E 2$ and $M 1$ transitions with increasing spin. Our analysis of the $B(E 2)$ and $B(M 1)$ patterns suggests that there are many examples in the odd-odd Cs nuclei that can be considered candidates to display chirality. In particular, the observed $B(E 2 ; I \rightarrow I-2)$ and $B(M 1 ; I \rightarrow I-1)$ intraband and interband transitions in the yrast and second-lowest bands of the ${ }^{128} \mathrm{Cs}$ nucleus show a definite staggering pattern as a function of the angular momentum [30]. Such a selection rule has been derived from symmetry considerations applied to a simple particle-rotor model [29]. Nevertheless, they can still be used to benchmark our calculations.

The predicted $B(E 2 ; I \rightarrow I-2)$ transition rates for most of the considered double-odd nuclei ${ }^{124-132} \mathrm{Cs}$ [see panels (a1) to (e1) on the left-hand side of Fig. 14] do not show any such staggering as the one that appears in the simplified model [29]. For the yrast band they evolve monotonically or stay rather constant with $I$. In some of the $B(E 2)$ transitions shown, at particular spin their values almost vanish, e.g., the $B\left(E 2 ; 16_{2}^{+} \rightarrow 14_{2}^{+}\right)$transition rate in ${ }^{128} \mathrm{Cs}$ in panel (c1). Particularly irregular $I$ dependence of the predicted $B(E 2)$ rates is found for the ${ }^{126} \mathrm{Cs}$ nucleus [see panel (b1)]. This is because certain mixing among states with a given spin tends to occur and, therefore, the assignments of the lowest states $I_{1}$ into the yrast band and of the second-lowest states $I_{2}$ into the side band are, in some cases, not adequate. In a number of the odd-odd Cs nuclei, however, a certain staggering pattern, similar to the one in the observed $B(M 1 ; I \rightarrow I-1)$ rates for ${ }^{128} \mathrm{Cs}$ [30], has been obtained in the calculated $B(M 1 ; I \rightarrow$ $I-1$ ) rates for both the intraband [middle panels (a2) to (e2) of Fig. 14] and interband [right panels (a3) to (e3)] transitions.
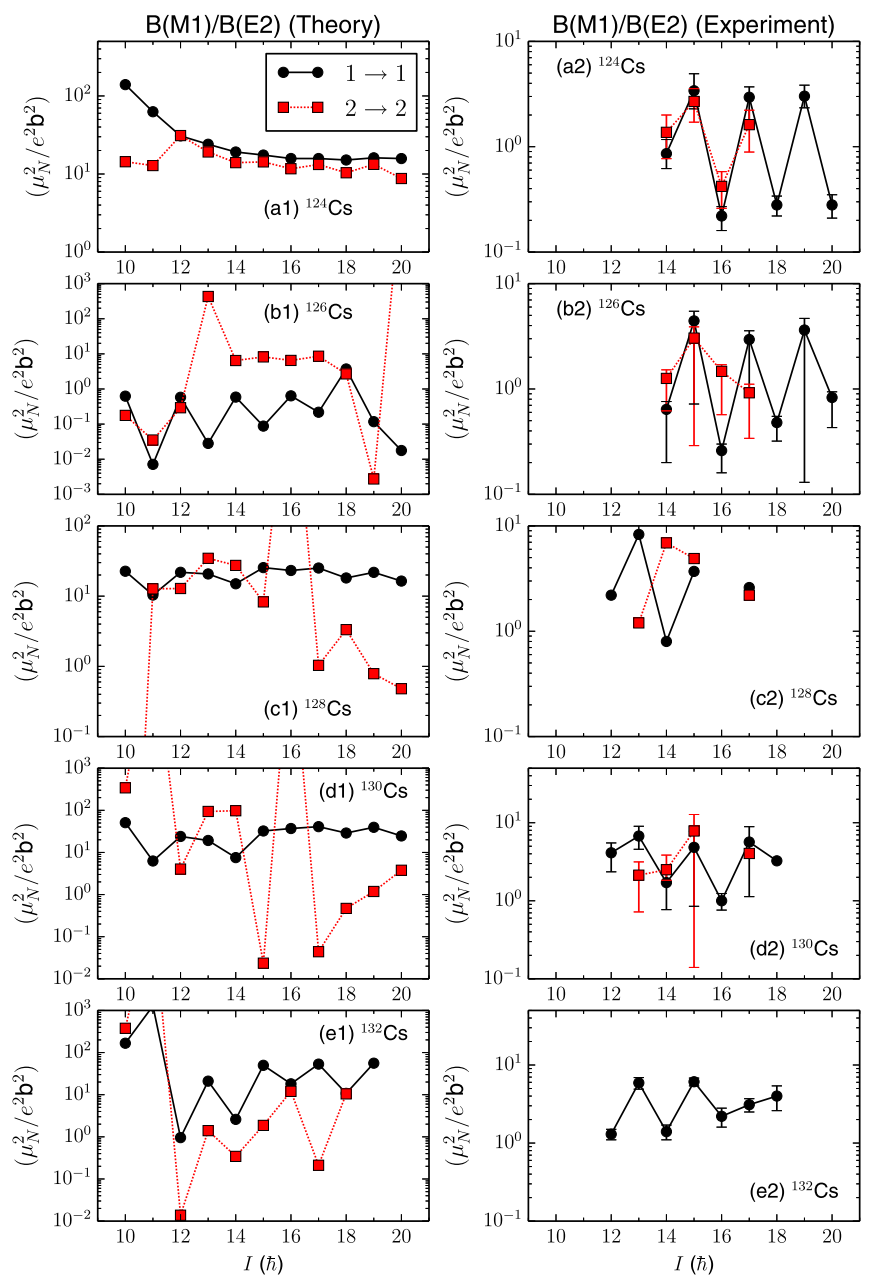

FIG. 15. The calculated and experimental values of the ratio $B(M 1 ; I \rightarrow I-1) / B(E 2 ; I \rightarrow I-2)$ (in $\mu_{N}^{2} / e^{2} \mathrm{~b}^{2}$ units) are plotted as a function of $I$ for the positive-parity yrast and side bands of the odd-odd nuclei ${ }^{124-132} \mathrm{Cs}$. The experimental data are taken from Refs. [57-62]. Note that calculated $B(M 1) / B(E 2)$ values lying well outside of the scale of the vertical axis are not shown.

As yet another indication of the chiral bands, we show in Fig. 15 the ratio of the calculated $B(M 1 ; I \rightarrow I-1)$ to $B(E 2 ; I \rightarrow I-2)$ rates of the yrast and side bands for all the odd-odd Cs nuclei. A number of experimental values for these quantities are available in Refs. [57-62]. Our results show a staggering pattern of the $B(M 1) / B(E 2)$ ratio as a function of angular momentum $I$ for both yrast and side bands in all the considered odd-odd Cs nuclei, except ${ }^{124} \mathrm{Cs}$ [panel (a1) of Fig. 15]. The theoretical values are consistent with the empirical trend (shown on the right-hand side of Fig. 15). However, the predicted $B(M 1) / B(E 2)$ values are much larger in magnitude and show a more irregular $I$ dependence than the experimental data. The quantitative disagreement can be expected from the behavior of the calculated $B\left(E 2 ; I_{1,2} \rightarrow(I-2)_{1,2}\right)$ [Figs. 14(a1)-14(e1)] and $B\left(M 1 ; I_{1,2} \rightarrow(I-1)_{1,2}\right)$ [Figs. 14(a2)-14(e2)] values as functions of $I$. 

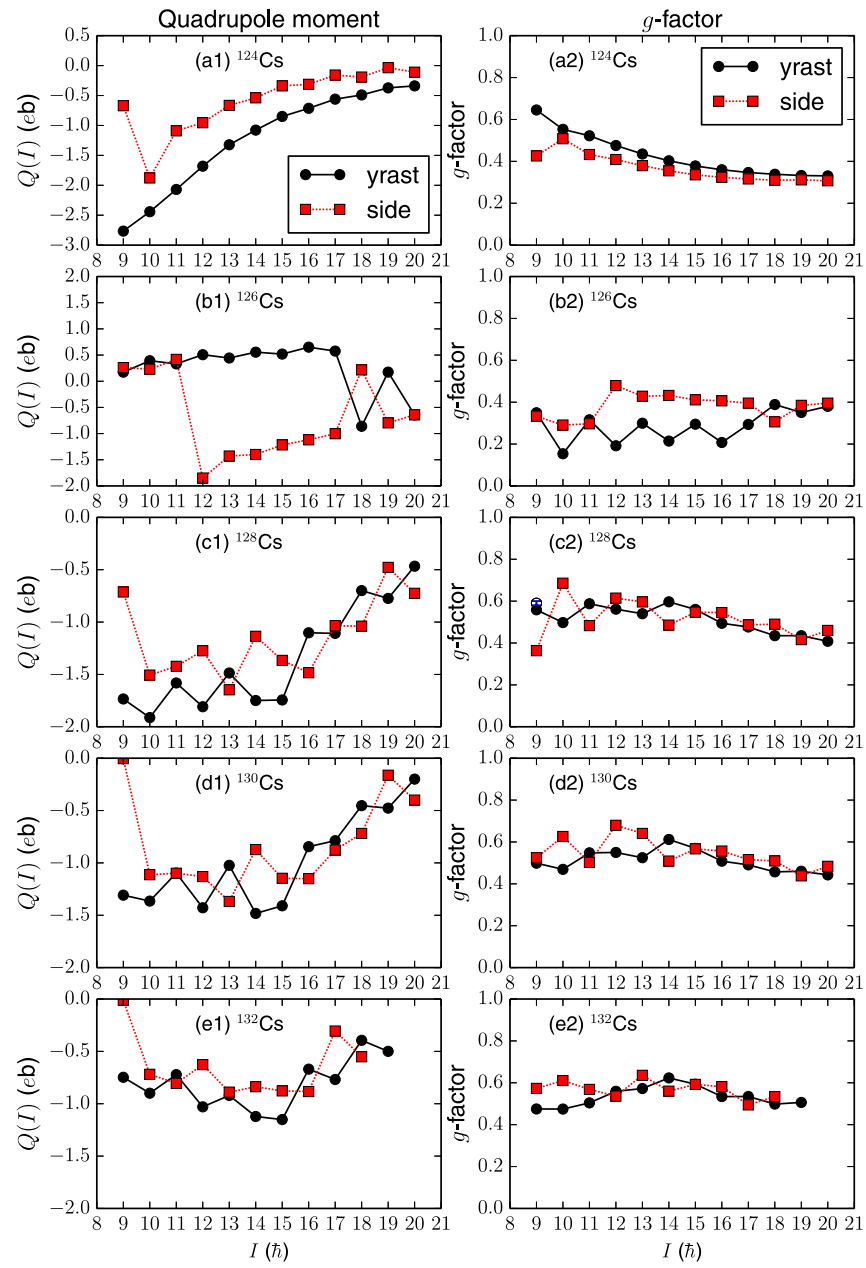

FIG. 16. Electric quadrupole moment $Q(I)$ (in $e$ b units) and $g$ factor as functions of angular momentum $I$ of the higher-spin yrast and side band states of the studied odd-odd Cs isotopes. In panel (c2), the experimental value for the $g$ factor of $+0.59 \pm 0.01$ for the yrast $9^{+}$state of ${ }^{128} \mathrm{Cs}[32]$ is shown as an open circle.

In order to examine whether the predicted yrast and side bands can be considered partners of the chiral doublet, we show in Fig. 16 the quadrupole moment $Q(I)$ (in $e$ b units) and the $g$ factor for the corresponding states in the considered odd-odd ${ }^{124-132} \mathrm{Cs}$ nuclei. The $Q(I)$ values are negative and decrease in magnitude with increasing spin. In addition, we have obtained similar $Q(I)$ values and $I$ dependence, for both bands. The above observation does not apply to the results for the ${ }^{126} \mathrm{Cs}$ nucleus in panel (b1). This is because, as we mentioned earlier, the states in the yrast and side bands for ${ }^{126} \mathrm{Cs}$ in particular, predicted in the present calculation, have different wave function contents. Namely, the side-band states are mainly composed of the $\left[\left(v h_{11 / 2}\right)^{-1} \otimes \pi h_{11 / 2}\right]^{(J)}$ singleparticle configuration, but this is not the case for those states in the yrast band. In all the odd-odd Cs nuclei, the $g$-factor values, depicted on the right-hand side of the same figure, are quite similar (around 0.5) for both bands. Note, that the $g$-factor obtained for the $I=9^{+}$yrast state for ${ }^{128} \mathrm{Cs}$ agrees well with the experimental value $(+0.59 \pm 0.01)$ [32].
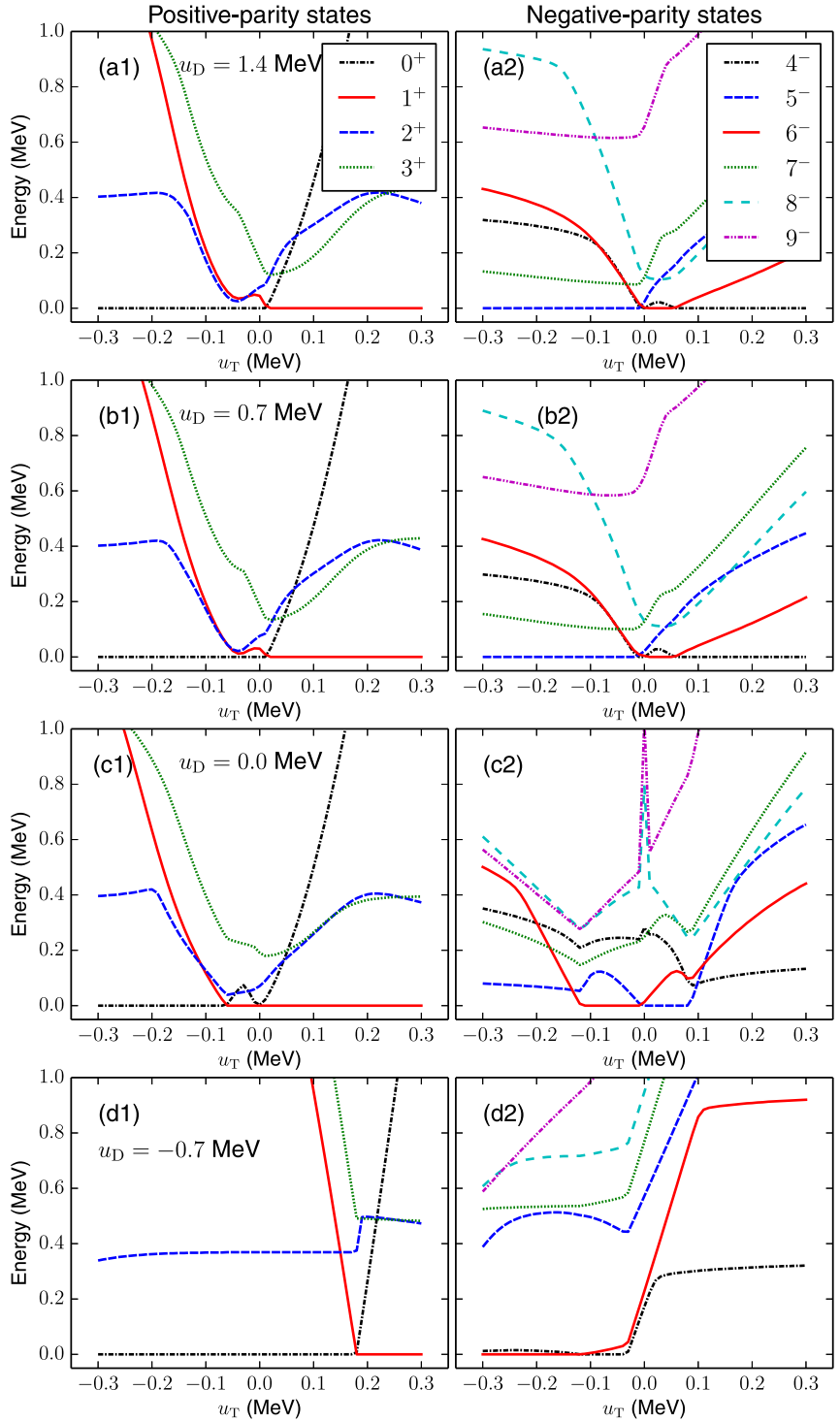

FIG. 17. Excitation energies of the low-lying positive- and negative-parity yrast states of the ${ }^{128} \mathrm{Cs}$ nucleus as functions of the parameters $u_{\mathrm{T}}$ in the cases of different values of the parameter $u_{\mathrm{D}}$, i.e., $u_{\mathrm{D}}=1.4 \mathrm{MeV}$ [panels (a1),(a2)], $0.7 \mathrm{MeV}$ [panels (b1),(b2)], $0.0 \mathrm{MeV}$ [panels (c1),(c2)], and $-0.7 \mathrm{MeV}$ [panels (d1),(d2)].

\section{Dependence on the residual neutron-proton interaction}

Finally we examine how the spectroscopic results from the IBFFM-2 depend on the choice of the strength parameters for the residual neutron-proton interaction $\hat{V}_{\text {res }}$ [see Eq. (7)]. In Fig. 17 we depict the evolution of the calculated excitation energies for a few low-lying positive-parity [panels (a1)-(d1) on the left-hand side of Fig. 17] and negative-parity [panels (a2)-(d2) on the right-hand side] yrast states of the ${ }^{128} \mathrm{Cs}$ nucleus as functions of the strength parameter for the tensor interaction $u_{\mathrm{T}}$ (in $\mathrm{MeV}$ ), in the cases of different values of the parameter for the delta interaction, i.e., $u_{\mathrm{D}}=1.4 \mathrm{MeV}$ [panels (a1),(a2)], $0.7 \mathrm{MeV}$ [panels (b1),(b2)], 0.0 MeV [panels (c1),(c2)], and $-0.7 \mathrm{MeV}$ [panels (d1),(d2)]. The calculated excitation energies, especially for the positive-parity ones, do 

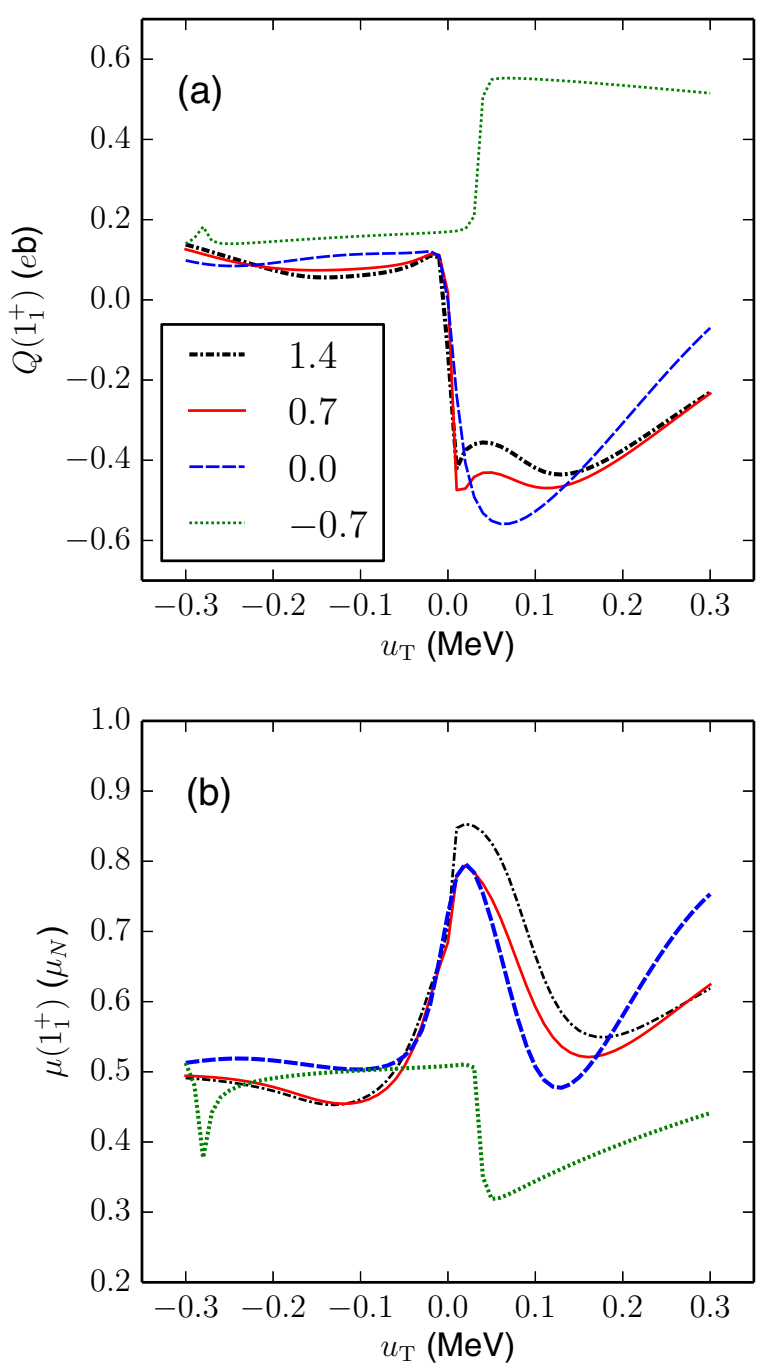

FIG. 18. The calculated quadrupole (a) and magnetic (b) moments of the $1_{1}^{+}$ground state for the ${ }^{128} \mathrm{Cs}$ nucleus as functions of the parameters $u_{\mathrm{T}}$ in the cases of different values of the parameter $u_{\mathrm{D}}$, i.e., $u_{\mathrm{D}}=1.4,0.7,0.0$, and $-0.7 \mathrm{MeV}$.

not seem to have a strong dependence on the parameter $u_{\mathrm{D}}$ for $u_{\mathrm{D}}>0 \mathrm{MeV}$, but are rather sensitive to $u_{\mathrm{T}}>0 \mathrm{MeV}$. Our choice, i.e., the value of the parameter $u_{\mathrm{T}}$, that is somewhere in the range $0<u_{\mathrm{T}}<0.05$, as well as the value of $u_{\mathrm{D}}$ to be approximately $0.7 \mathrm{MeV}$, seems to be optimal for reproducing the ground-state spin for both the positive and negative parities, i.e., $I=1_{1}^{+}$and $6_{1}^{-}$, respectively.

Similarly, in Fig. 18 we plot the calculated quadrupole $Q\left(1_{1}^{+}\right)$and magnetic $\mu\left(1_{1}^{+}\right)$moments for the ground state $1_{1}^{+}$ of the ${ }^{128} \mathrm{Cs}$ nucleus as functions of the strength parameter $u_{\mathrm{T}}$, in the cases of different values of the parameter $u_{\mathrm{D}}=1.4,0.7$, 0.0 , and $-0.7 \mathrm{MeV}$. Both the $Q\left(1_{1}^{+}\right)$and $\mu\left(1_{1}^{+}\right)$values depend somewhat largely on the parameter $u_{\mathrm{T}}$ with $u_{\mathrm{T}}>0 \mathrm{MeV}$, but are much less sensitive to the parameter $u_{\mathrm{D}}>0 \mathrm{MeV}$. The chosen value for the tensor interaction strength $u_{\mathrm{T}}$ of 0.02 $\mathrm{MeV}$ gives both the $Q\left(1_{1}^{+}\right)$and $\mu\left(1_{1}^{+}\right)$values close to the corresponding experimental data, $Q\left(1_{1}^{+}\right)=-0.570 \pm 0.08 \mathrm{eb}$ and $\mu\left(1_{1}^{+}\right)=+0.974 \pm 0.005 \mu_{N}$, respectively.
Similar parameter dependences of the excitation energies and moments have been obtained for the other odd-odd Cs studied here. In principle, one could use values of the $u_{\mathrm{D}}$ and $u_{\mathrm{T}}$ parameters that are different from nucleus to nucleus and/or between both parities. We have used the fixed values for the $u_{\mathrm{D}}$ and $u_{\mathrm{T}}$ parameters only for the sake of simplicity. Nevertheless, we consider the chosen values of the $u_{\mathrm{D}}$ and $u_{\mathrm{T}}$ strength parameters to be realistic in the sense that the overall description of the energy levels of the low-lying low-spin states for the studied odd-odd nuclei is reasonable.

\section{SUMMARY AND CONCLUDING REMARKS}

The spectroscopic properties of the odd-odd nuclei ${ }^{124-132} \mathrm{Cs}$ have been analyzed using the interacting bosonfermion-fermion (IBFFM-2) framework with microscopic input from mean field calculations with the Gogny-D1M energy density functional. The $(\beta, \gamma)$-deformation energy surface for even-even boson-core $\mathrm{Xe}$ isotopes as well as single-particle energies and occupation probabilities of unpaired nucleons in odd- $N$ Xe, odd- $Z$ Cs and odd-odd Cs nuclei obtained from the mean field calculation are used to build, via a mapping procedure, the corresponding IBFFM-2 Hamiltonian. In its current implementation, the method still requires a few coupling constants of the boson-fermion and residual neutron-proton interactions to be fitted to the experiment. The diagonalization of the corresponding IBFFM-2 Hamiltonian provides wave functions, energy levels, as well as other spectroscopic properties such as $E 2$ and $M 1$ transition rates.

It has been shown that the (mapped) IBFFM-2 model describes reasonably well both the positive- and negative-parity low-lying low-spin states of the considered odd-odd Cs nuclei, especially in the case of ${ }^{124,126,128}$ Cs. This is a remarkable result, considering the significant reduction of parameters with respect to previous IBFFM calculations. However, in some of the odd-odd nuclei (e.g., in ${ }^{132} \mathrm{Cs}$ ) the ordering of both the positive- and negative-parity levels close to the ground state could not be correctly reproduced by our calculations. Some possible explanations for this failure are the limited number of active bosons in the even-even core near the shell closure; the possibility that the adopted single-particle energies and occupation numbers provided by the Gogny-D1M HFB approach may not be realistic enough; and finally the use of fixed strengths for the whole isotopic chain for the residual neutron-proton interaction in the IBFFM-2 Hamiltonian.

We have also studied the band structure of the higher-spin positive-parity states in the considered odd-odd Cs nuclei. Our calculations provide a reasonable quantitative description of the excitation energies of these bands up to $I \approx 20^{+}$except for the excitation energy of the bandheads of ${ }^{126} \mathrm{Cs}$ which are overestimated. We have identified many of the double-odd Cs nuclei as good candidate for the existence of chiral doublet bands. In particular, the calculated $B(M 1 ; I \rightarrow I-1)$ transition rates exhibit staggering patterns with increasing angular momentum. This result agrees well with the selection rule derived by simple symmetry considerations [29]. All in all, the results of this study suggest that the employed theoretical methods can be potentially used to describe even such a type of nuclear excitation as chirality. 


\section{ACKNOWLEDGMENTS}

The work of K.N. is financed within the Tenure Track Pilot Program of the Croatian Science Foundation and the École Polytechnique Fédérale de Lausanne and the Project TTP-2018-07-3554 Exotic Nuclear Structure and Dynamics, with funds of the Croatian-Swiss Research Program, the
Croatian Science Foundation, and École Polytechnique Fédérale de Lausanne under the Swiss-Croatian Corporation Program No. TTP-2018-07-3554. The work of L.M.R. was supported by the Spanish Ministry of Economy and Competitiveness (MINECO) Grants No. FPA2015-65929-MINECO and No. FIS2015-63770-MINECO.
[1] P. Ring and P. Schuck, The Nuclear Many-Body Problem (Springer-Verlag, Berlin, 1980).

[2] M. Bender, P.-H. Heenen, and P.-G. Reinhard, Rev. Mod. Phys. 75, 121 (2003).

[3] L. M. Robledo, T. R. Rodríguez, and R. R. Rodríguez-Guzmán, J. Phys. G: Nucl. Part. Phys. 46, 013001 (2019).

[4] B. Bally, B. Avez, M. Bender, and P.-H. Heenen, Phys. Rev. Lett. 113, 162501 (2014).

[5] M. Borrajo and J. L. Egido, Eur. Phys. J. A 52, 277 (2016).

[6] J. Dobaczewski, A. Afanasjev, M. Bender, L. Robledo, and Y. Shi, Nucl. Phys. A 944, 388 (2015), Special Issue on Superheavy Elements.

[7] E. Caurier, G. Martínez-Pinedo, F. Nowack, A. Poves, and A. P. Zuker, Rev. Mod. Phys. 77, 427 (2005).

[8] K. Nomura, R. Rodríguez-Guzmán, and L. M. Robledo, Phys. Rev. C 99, 034308 (2019).

[9] J. Decharge, M. Girod, and D. Gogny, Phys. Lett. B 55, 361 (1975).

[10] S. Goriely, S. Hilaire, M. Girod, and S. Péru, Phys. Rev. Lett. 102, 242501 (2009).

[11] F. Iachello and A. Arima, The Interacting Boson Model (Cambridge University Press, Cambridge, 1987).

[12] F. Iachello and O. Scholten, Phys. Rev. Lett. 43, 679 (1979).

[13] O. Scholten, Prog. Part. Nucl. Phys. 14, 189 (1985).

[14] F. Iachello and P. Van Isacker, The Interacting Boson-Fermion Model (Cambridge University Press, Cambridge, 1991).

[15] S. Brant, V. Paar, and D. Vretenar, Z. Phys. A 319, 355 (1984).

[16] R. Casten and P. V. Brentano, Phys. Lett. B 152, 22 (1985).

[17] A. Sevrin, K. Heyde, and J. Jolie, Phys. Rev. C 36, 2631 (1987).

[18] J. Yan, O. Vogel, P. von Brentano, and A. Gelberg, Phys. Rev. C 48, 1046 (1993).

[19] O. Vogel, P. Van Isacker, A. Gelberg, P. von Brentano, and A. Dewald, Phys. Rev. C 53, 1660 (1996).

[20] T. Mizusaki and T. Otsuka, Prog. Theor. Phys. Suppl. 125, 97 (1996).

[21] N. Yoshinaga and K. Higashiyama, Phys. Rev. C 69, 054309 (2004).

[22] Z. P. Li, T. Nikšić, D. Vretenar, and J. Meng, Phys. Rev. C 81, 034316 (2010).

[23] K. Nomura, N. Shimizu, D. Vretenar, T. Nikšić, and T. Otsuka, Phys. Rev. Lett. 108, 132501 (2012).

[24] P. Cejnar, J. Jolie, and R. F. Casten, Rev. Mod. Phys. 82, 2155 (2010).

[25] R. F. Casten and N. V. Zamfir, Phys. Rev. Lett. 85, 3584 (2000).

[26] L. Coquard, N. Pietralla, T. Ahn, G. Rainovski, L. Bettermann, M. P. Carpenter, R. V. F. Janssens, J. Leske, C. J. Lister, O. Möller, W. Rother, V. Werner, and S. Zhu, Phys. Rev. C 80, 061304(R) (2009).

[27] F. Iachello, Phys. Rev. Lett. 85, 3580 (2000).

[28] S. Frauendorf and J. Meng, Nucl. Phys. A 617, 131 (1997).
[29] T. Koike, K. Starosta, and I. Hamamoto, Phys. Rev. Lett. 93, 172502 (2004).

[30] E. Grodner, J. Srebrny, A. A. Pasternak, I. Zalewska, T. Morek, C. Droste, J. Mierzejewski, M. Kowalczyk, J. Kownacki, M. Kisieliński et al., Phys. Rev. Lett. 97, 172501 (2006).

[31] K. Starosta and T. Koike, Phys. Scr. 92, 093002 (2017).

[32] E. Grodner, J. Srebrny, C. Droste, L. Próchniak, S. G. Rohoziński, M. Kowalczyk, M. Ionescu-Bujor, C. A. Ur, K. Starosta, T. Ahn et al., Phys. Rev. Lett. 120, 022502 (2018).

[33] S. Brant, D. Vretenar, and A. Ventura, Phys. Rev. C 69, 017304 (2004).

[34] N. Yoshinaga and K. Higashiyama, Eur. Phys. J. A 30, 343 (2006).

[35] K. Higashiyama, N. Yoshinaga, and K. Tanabe, Phys. Rev. C 72, 024315 (2005).

[36] K. Higashiyama and N. Yoshinaga, Eur. Phys. J. A 33, 355 (2007).

[37] K. Higashiyama and N. Yoshinaga, Phys. Rev. C 88, 034315 (2013).

[38] L. Zuffi, S. Brant, and N. Yoshida, Phys. Rev. C 68, 034308 (2003).

[39] S. Brant, N. Yoshida, and L. Zuffi, Phys. Rev. C 74, 024303 (2006).

[40] E. Mardones, J. Barea, C. E. Alonso, and J. M. Arias, Phys. Rev. C 93, 034332 (2016).

[41] J. Engel and J. Menéndez, Rep. Prog. Phys. 80, 046301 (2017).

[42] J. Arias, C. Alonso, and R. Bijker, Nucl. Phys. A 445, 333 (1985).

[43] T. Otsuka, A. Arima, and F. Iachello, Nucl. Phys. A 309, 1 (1978).

[44] J. M. Arias, C. E. Alonso, and M. Lozano, Phys. Rev. C 33, 1482 (1986).

[45] N. Yoshida and F. Iachello, Prog. Theor. Exp. Phys. 2013, 043D01 (2013).

[46] I. Morrison, A. Faessler, and C. Lima, Nucl. Phys. A 372, 13 (1981).

[47] K. Nomura, N. Shimizu, and T. Otsuka, Phys. Rev. Lett. 101, 142501 (2008).

[48] K. Nomura, N. Shimizu, and T. Otsuka, Phys. Rev. C 81, 044307 (2010).

[49] J. N. Ginocchio and M. W. Kirson, Nucl. Phys. A 350, 31 (1980).

[50] K. Nomura, T. Nikšić, and D. Vretenar, Phys. Rev. C 93, 054305 (2016).

[51] K. Nomura, R. Rodríguez-Guzmán, and L. M. Robledo, Phys. Rev. C 96, 014314 (2017).

[52] N. Yoshida and A. Arima, Phys. Lett. B 164, 231 (1985).

[53] Brookhaven National Nuclear Data Center, http://www.nndc. bnl.gov 
[54] K. Nomura, R. Rodríguez-Guzmán, and L. M. Robledo, Phys. Rev. C 96, 064316 (2017).

[55] X.-F. Li, Y.-J. Ma, Y.-Z. Liu, J.-B. Lu, G.-Y. Zhao, L.-C. Yin, R. Meng, Z.-L. Zhang, L.-J. Wen, X.-H. Zhou et al., Eur. Phys. J. A 17, 523 (2003).

[56] N. Stone, At. Data Nucl. Data Tables 90, 75 (2005).

[57] B. Xiong and Y. Wang, At. Data Nucl. Data Tables 125, 193 (2019).

[58] A. Gizon, J. Timár, J. Gizon, B. Weiss, D. Barnéoud, C. Foin, J. Genevey, F. Hannachi, C. Liang, A. Lopez-Martens et al., Nucl. Phys. A 694, 63 (2001).
[59] S. Wang, Y. Liu, T. Komatsubara, Y. Ma, and Y. Zhang, Phys. Rev. C 74, 017302 (2006).

[60] E. S. Paul, D. B. Fossan, Y. Liang, R. Ma, and N. Xu, Phys. Rev. C 40, 619 (1989).

[61] A. J. Simons, P. Joshi, D. G. Jenkins, P. M. Raddon, R. Wadsworth, D. B. Fossan, T. Koike, C. Vaman, K. Starosta, E. S. Paul et al., J. Phys. G: Nucl. Part. Phys. 31, 541 (2005).

[62] G. Rainovski, E. S. Paul, H. J. Chantler, P. J. Nolan, D. G. Jenkins, R. Wadsworth, P. Raddon, A. Simons, D. B. Fossan, T. Koike et al., Phys. Rev. C 68, 024318 (2003). 